\title{
Ordering variable for parton showers
}

\author{
Zoltán Nagy ${ }^{a}$ and Davison E. Soper ${ }^{b}$ \\ ${ }^{a} D E S Y$, \\ Notkestrasse 85, 22607 Hamburg, Germany \\ ${ }^{b}$ Institute of Theoretical Science, University of Oregon, \\ Eugene, OR 97403-5203, U.S.A. \\ E-mail: Zoltan.Nagy@desy.de, soper@uoregon.edu
}

ABSTRACT: The parton splittings in a parton shower are ordered according to an ordering variable, for example the transverse momentum of the daughter partons relative to the direction of the mother, the virtuality of the splitting, or the angle between the daughter partons. We analyze the choice of the ordering variable and conclude that one particular choice has the advantage of factoring softer splittings from harder splittings graph by graph in a physical gauge.

Keywords: QCD Phenomenology, Monte Carlo Simulations

ARXIV EPRINT: 1401.6366 


\section{Contents}

1 Introduction 1

2 Ordering variable choices in other parton shower algorithms 2

2.1 Angular ordering 4

2.2 Hardness orderings and factorization 5

2.3 Choices for the hardness parameter 6

2.4 Definitions of transverse momentum $\quad 7$

$\begin{array}{ll}2.5 & \text { Summing large logarithms }\end{array}$

3 Definition of ordering variable in Deductor $\quad 10$

$\begin{array}{ll}3.1 \text { Shower time for final state splittings } & 10\end{array}$

$\begin{array}{lll}3.2 & \text { Shower time and physical time } & 14\end{array}$

$\begin{array}{lll}3.3 & \text { Shower time for initial state splittings } & 15\end{array}$

$\begin{array}{lll}3.4 & \text { Interference graphs } & 17\end{array}$

4 Consequences for final state splittings $\quad 20$

4.1 Kinematics 20

$\begin{array}{lll}4.2 & \text { A specific case } & 22\end{array}$

5 Consequences for initial state splittings $\quad \mathbf{2 3}$

5.1 Kinematics 24

$\begin{array}{ll}5.2 & \text { Evolution of the gluon virtualities }\end{array}$

$\begin{array}{ll}5.3 \text { Momentum conservation } & 26\end{array}$

$\begin{array}{ll}5.4 \text { Dynamical regimes } & 28\end{array}$

$\begin{array}{ll}5.5 \text { A numerical investigation } & 30\end{array}$

6 Ending the shower $\quad 31$

$\begin{array}{lll}7 & \text { Conclusions } & 32\end{array}$

$\begin{array}{ll}\text { A Momentum conservation for initial state splitting } & 33\end{array}$

\section{Introduction}

In a companion paper [1], we have introduced a parton shower event generator, DEDUCTOR [2], based on our earlier work [3, 4]. The corresponding shower algorithm is designed to be suitable for an improved treatment of spin, as described in ref. [5], and for an improved treatment of color, as described in ref. [6]. This shower generator contains features that differ from other parton shower event generators even when one uses the leading color 
approximation and averages over spins, as we do in ref. [1]. One of these features is that the algorithm uses non-zero masses for initial state partons, which requires modified evolution equations for the parton distribution functions, as described in a separate companion paper [7]. The second feature is the choice of shower evolution variable, which is the subject of this paper.

In a parton shower event generator, when a parton labelled $i$ splits to two partons, one typically assigns a variable $V_{i}^{2}$ to the splitting, where $V_{i}^{2}$ is a function of the momenta of the mother parton and its daughters. The purpose of defining $V_{i}^{2}$ is to order splittings within the shower: if the splitting of parton $i$ comes before the splitting of parton $j$ then $V_{i}^{2}>V_{j}^{2}$. When $V_{i}^{2}$ has dimensions of mass squared, one can define a dimensionless shower time $t_{i} \propto \log \left(Q_{0}^{2} / V_{i}^{2}\right)$ where $Q_{0}^{2}$ denotes the scale of the hard interaction that initiates the shower. Then $t$ increases as the shower progresses.

In most cases ( $k_{\mathrm{T}}$-ordering, virtuality ordering, but not angular ordering), $V_{i}^{2}$ is a measure of the hardness of the splitting: $V_{i}^{2} \rightarrow 0$ when the angle between the daughter partons approaches zero or when the momentum of one of the daughter partons approaches zero. The ordering variable in DEDuctor is hardness based in this sense.

Our purpose in this paper is to explain what ordering variable $V_{i}^{2}$ appears in DeDUCTOR and why we made this particular choice. Before explaining our choice, we provide in section 2 some background information on choices for the ordering variable that are used in current parton shower event generators. We present the definition of $V_{i}^{2}$ used in DEDUCTOR in section 3. In section 4, we present a case study for final state splittings of how the choice of ordering variable affects the shower histories generated. Then, in section 5, we show in initial state splittings that the ordering used DEDUCTOR allows a wider range of initial state radiation as a function of $p_{\mathrm{T}}$ and rapidity than would be produced with virtuality ordering. There is a short discussion of ending the perturbative shower in section 6. Some conclusions follow in section 7. There is one appendix, A, on momentum conservation in initial state splittings.

\section{Ordering variable choices in other parton shower algorithms}

A parton shower event generator for hadron-hadron scattering is a computer program that generates final states starting with some hard process. For instance, the hard process could be Higgs boson production, W-boson production, or just large transverse momentum quark-quark scattering via gluon exchange. The program then generates complete events, consisting of the momenta and flavors of a number of final state particles. The idea is that the weight for an event times the probability that the event is generated is an approximation to the cross section for producing that event. ${ }^{1}$ In the event generators HeRwig [8, 9], Pythia [10, 11], and Sherpa [12, 13], the final state consists of hadrons. Our event generator, DEDuctor, does not include a hadronization stage, so the final states consist of partons. Our discussion in this paper concerns only the parton shower part of an event generator, not the model for hadronization.

\footnotetext{
${ }^{1}$ Sometimes, the weights are all the same. To keep the language simple, we refer to just probabilities rather than probabilities times weights.
} 


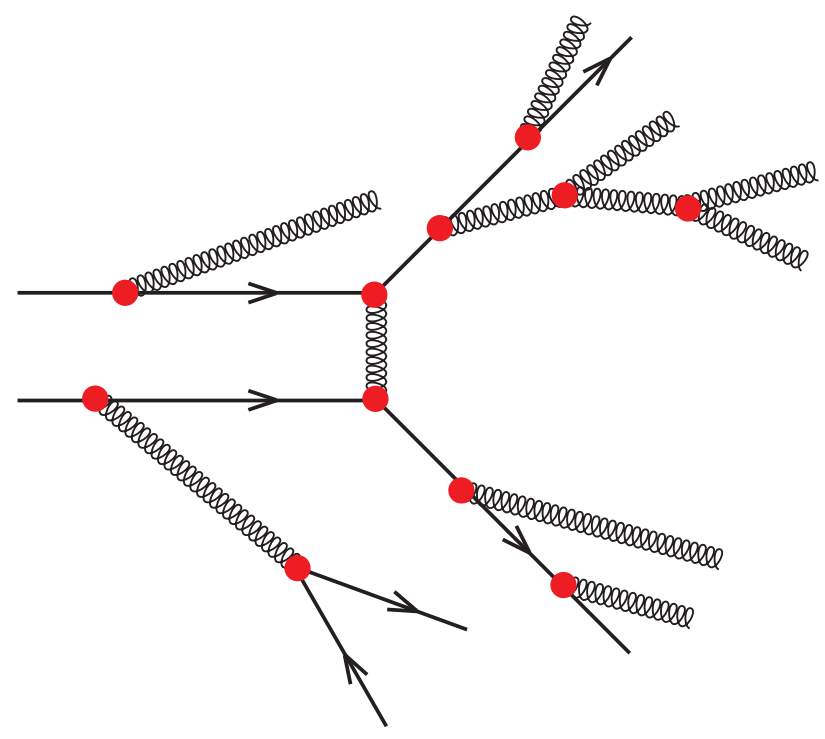

Figure 1. A possible shower history. The hard interaction is quark-quark scattering via gluon exchange. Both the initial state quarks and the final state quarks can emit gluons. Shower time increases as one moves away from the hard interaction. Parton splittings at later shower time are softer.

In a parton shower in the sense used in this paper, one parton splits into two partons according to a splitting probability based on QCD matrix elements. A parton shower can also be organized as an antenna shower, as in ARIADNe [14-17] and Vincia [18]. A "dipole antenna" with contributions from two partons splits into two dipole antennas involving a total of three partons. Discussion of ordering variables needed for this sort of shower organization is beyond the scope of this paper.

For each generated partonic event, there is a shower history, $H$, as in figure 1 . The shower history has the appearance of a Feynman diagram. However, the connection between the shower history diagram and Feynman diagrams may be quite indirect. The shower history for a given simulated event tells us how the event was generated.

First, the generator creates the momenta of the partons involved in the hard scattering with a probability proportional to the squared matrix element for the hard scattering times the parton distribution functions for the initial state partons.

The program then successively generates splittings of the partons. A final state parton can split into two daughters. Similarly, an initial state parton can split into two daughters, one of which is a newly created final state parton and one of which is a new initial state parton. In either case, the daughter momenta for a given splitting are drawn from a probability distribution that depends on the system configuration just before the splitting. The splitting probabilities are based on the QCD lagrangian but depend also on just how the shower is organized.

Parton shower event generators typically have a scheme for ordering successive splittings. When a parton labelled $i$ splits to two partons, one typically assigns a variable 

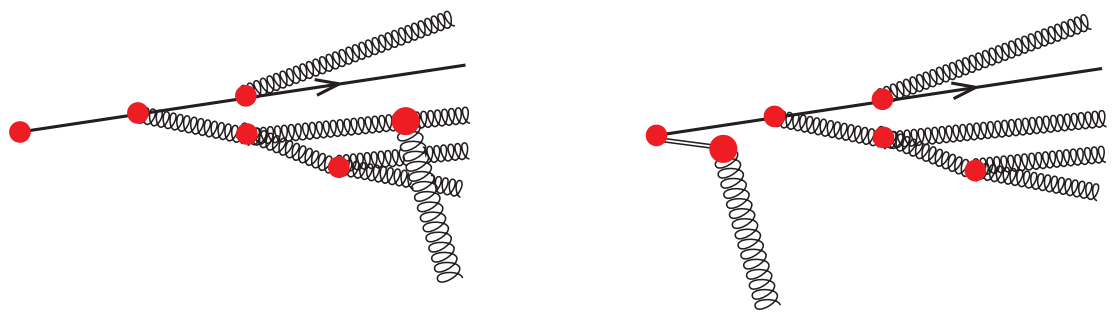

Figure 2. The effect of coherence in wide angle gluon emissions. In the graph on the left, a soft, wide angle gluon is emitted from one of five partons that are part of a narrow jet. The sum of these graphs is approximately equal to the graph on the right in which the soft gluon attaches to an eikonal line (with Feynman rule $\left.\left(\varepsilon \cdot u_{\mathrm{J}} / q \cdot u_{\mathrm{J}}\right) t_{K}^{a}\right)$ moving in the jet direction.

$V_{i}^{2}$ to the splitting, where $V_{i}^{2}$ is a function of the momenta of the mother parton and its daughters. If the splitting of parton $i$ comes before the splitting of parton $j$ then $V_{i}^{2}>V_{j}^{2}$. This paper concerns a particular choice of the function $V_{i}^{2}$. Before presenting our choice, it seems worthwhile to provide some background concerning commonly used choices.

\subsection{Angular ordering}

One ordering principle was introduced in refs. [19, 20] and forms the basis for HeRwiG [8, 9]. The essential insight is illustrated in figure 2. Imagine that several massless, on-shell partons form a narrow angle jet in the sense that their three-momenta $\vec{p}_{l}$ are all large and very nearly parallel. Now let a gluon with momentum $q$ be emitted from the partons in the jet. We suppose that the gluon is soft, $|\vec{q}| \ll\left|\vec{p}_{l}\right|$ and that the angle between $\vec{q}$ and the $\vec{p}_{l}$ is much larger than the angles between the $\vec{p}_{l}$. Then the amplitude for emission from parton $l$ is approximately

$$
\mathcal{A}_{l}=\frac{\varepsilon \cdot u_{\mathrm{J}}}{q \cdot u_{\mathrm{J}}} t_{(l)}^{a}
$$

where $u_{\mathrm{J}}$ is a lightlike vector in the common direction of the $p_{l}, q$ is the momentum of the soft gluon, $\varepsilon$ is its polarization, and $a$ is its color index. The color matrix $t^{a}$ is inserted onto the color line representing parton $l$. Summing over all of the partons $l$ in the jet, we have

$$
\sum_{l} \mathcal{A}_{l}=\frac{\varepsilon \cdot u_{\mathrm{J}}}{q \cdot u_{\mathrm{J}}} \sum_{l} t_{(l)}^{a}
$$

The crucial point is that the only dependence of $\mathcal{A}_{l}$ on $l$ is through the color matrix $t_{(l)}^{a}$. One calls this phenomenon coherence. It is analogous to probing a nucleus with a soft electromagnetic field: the probe detects only the total electric charge of the nucleus, not the individual charges of the nucleons. In the present case, if the entire jet was descended from one mother parton with index $K$, then

$$
\sum_{l} t_{(l)}^{a}=t_{K}^{a}
$$

Thus the entire amplitude is given by

$$
\sum_{l} \mathcal{A}_{l}=\frac{\varepsilon \cdot u_{\mathrm{J}}}{q \cdot u_{\mathrm{J}}} t_{K}^{a}
$$


It is just as if the soft gluon had been emitted from the mother parton, treating the mother parton momentum as being proportional to $u_{\mathrm{J}}$.

We see that when our soft gluon momentum makes an angle $\theta$ with $\vec{n}_{\mathrm{J}}$ that is larger than any of the internal angles in the jet, it is as if the soft gluon were emitted first from a lightlike parton. This suggests that in generating this event, we can simply emit the large angle gluon first. That is, we can order emissions in order of decreasing angle: $V^{2} \propto \theta^{2}$. (See section 6.1 of ref. [9] for the exact definitions.)

This still works for collinear splittings, in which the angle is small and neither daughter parton is much softer than the other. We simply need to adjust the splitting function appropriately.

The coherence argument given above seems so powerful that one may wonder how a parton shower based on anything other than angular ordering can represent the proper physics. To see how the same physics can appear in another guise, consider the product of the amplitude for emitting our soft gluon and the corresponding complex conjugate amplitude,

$$
\left(\sum_{l} \mathcal{A}_{l}\right)\left(\sum_{k} \mathcal{A}_{k}\right)^{*}=\sum_{l k} \mathcal{A}_{l} \mathcal{A}_{k}^{*} .
$$

We get the correct result if we include all of the interference terms, those with $l \neq k$. A so-called "dipole shower" includes the interference terms, at least approximately. In SHERPA and in the final state shower of PyтнiA, the interference terms are included in the "leading color" approximation, in which terms of relative order $1 / N_{\mathrm{c}}^{2}$ are dropped (with $N_{\mathrm{c}}=3$ being the number of colors). Thus most of the good features of angular ordering are retained in dipole showers with other ordering variables. ${ }^{2}$

Is there any disadvantage to angular ordering? One may note that the derivation above assumed that the angles were strongly ordered: $\theta\left(q, p_{l}\right) \gg \theta\left(p_{l}, p_{k}\right)$. If the angles are ordered, but not strongly ordered, then we have made an approximation. As argued in refs. $[19,20]$, the approximation becomes exact if we average over suitable azimuthal angles. However, if we want to know the distribution of the emissions as a function of the azimuthal angles, then the result calculated according to eq. (2.4) is only approximate and has large fractional errors.

\subsection{Hardness orderings and factorization}

In many parton shower algorithms, the ordering variable $V^{2}$ is a measure of the hardness of the splitting: $V^{2} \rightarrow 0$ when the angle between the daughter partons approaches zero or when the momentum of one of the daughter partons approaches zero. That is, if $V^{2}$ is fixed at a positive value, then the splitting cannot reach either the collinear singularity or the soft singularity of the corresponding QCD matrix element. The ordering variable in DEDUCTOR is hardness based in this sense.

There is a physics reason for choosing a hardness based ordering parameter. The reason is related to the phenomenon of factorization. Factorization is applied to suitable

\footnotetext{
${ }^{2}$ Our program, Deductor can go beyond the leading color approximation, although it still does not do color exactly.
} 
inclusive observables such a jet cross section or the cross section to produce a very heavy particle. The observable is characterized by a large squared momentum scale, typically called $Q^{2}$. In a calculation of the observable, we choose a factorization scale, typically called $\mu^{2}$. (Here we identify the factorization and renormalization scales.) Factorization means that we can write the corresponding cross section as a convolution of three factors: a calculable partonic cross section $d \hat{\sigma}\left(\mu^{2}\right)$ and two parton distribution functions $f\left(x, \mu^{2}\right)$ for the two incoming hadrons. The scale parameter in the parton distribution functions is $\mu^{2}$ and the calculation of the partonic cross section explicitly involves $\mu^{2}$. Usually, we pick $\mu^{2}=Q^{2}$, but that choice is not necessary. If our calculation is accurate to order $\alpha_{\mathrm{s}}^{N}$, then the result of the calculation is independent of $\mu^{2}$ to that order.

Now suppose that we calculate the same observable with very large scale $Q^{2}$, but using a parton shower calculation. In the context of this calculation, the shower hardness parameter $V^{2}$ corresponds to $\mu^{2}$. If we like, we can choose $V^{2}$ to be much less than the hardest scale, $Q^{2}$. The parton shower run to a given value of $V^{2}$ constitutes an approximate calculation of $d \hat{\sigma}\left(V^{2}\right)$. The parton shower result then depends on parton distribution functions $f\left(x, \mu^{2}\right)$ evaluated at scale $\mu^{2} \sim V^{2}$. (We discuss this in some detail in ref. [7].) Of course, if what we want is a calculation using a parton shower of a single observable with a very large scale $Q^{2}$, we do not need to choose $V^{2}$ much smaller than $Q^{2}$. However, we may be interested in other observables at the same time.

We can imagine an observable that has a resolution scale $Q_{1}^{2}<Q^{2}$. With this observable, we see what happens at space-time separations from the hard interaction that are smaller than or of order $1 / Q_{1}$. Anything that happens at larger space-time separations is "unresolvable." To measure such an observable, we need to run the parton shower to a hardness value $V^{2} \sim Q_{1}^{2}$. When we use an observable that has a resolution down to softer interactions $Q_{1}^{2}$, we see more detail than we did with our original observable. The greater detail develops at greater space-time separations than the original $1 / Q$. The parton shower algorithm allows us to see more detail if we generate more splittings that are softer and softer.

We have compared an ordinary perturbative calculation to a parton shower calculation. Note that the treatment of virtual graphs in these two types of calculations is different. In a parton shower, the virtual graphs plus the parts of the integration of real graphs corresponding to soft momenta become the Sudakov exponent.

Thus our view is that the shower evolution parameter characterizing the hardness of splittings corresponds to the factorization scale and shower evolution is equivalent to the use of the renormalization group to change the factorization scale. We should emphasize that this view of shower evolution extends the idea of factorization way beyond anything that has been proved.

\subsection{Choices for the hardness parameter}

There are more than one possibilities for $V^{2}$. Suppose that the daughter partons in a final state splitting have momenta $p_{i}$ and $p_{j}$. To keep our discussion simple, let us assume that all partons are massless. One possibility for the hardness parameter is the virtuality $V^{2}=\left(p_{i}+p_{j}\right)^{2}$. This choice was made in the earliest versions of PythiA. It has the evident advantages of being simple and Lorentz invariant. It is instructive to write the 
virtuality for the case in which the partons are part of a narrow jet, so that all energies are large and all angles are small. In this case, we can let $E=E_{i}+E_{j}$ be the energy of the mother parton and define momentum fractions $z=E_{i} / E,(1-z)=E_{j} / E$. Let $\theta_{i j}$ be the angle between the two partons. Then

$$
\left(p_{i}+p_{j}\right)^{2} \approx E^{2} z(1-z) \theta_{i j}^{2},
$$

Another possibility for the hardness parameter in the splitting is square of the transverse momentum $k_{\mathrm{T}}$ of either of the partons relative to the mother parton direction. In the narrow jet limit, this is

$$
k_{\mathrm{T}}^{2} \approx E^{2} z^{2}(1-z)^{2} \theta_{i j}^{2} .
$$

Since $k_{\mathrm{T}}^{2}$ is not Lorentz invariant, there is some ambiguity in how it is defined away from the narrow jet limit. Different shower algorithms make different choices, as we will explain below. We note that $\left(p_{i}+p_{j}\right)^{2}$ and $k_{\mathrm{T}}^{2}$ share the property that they vanish in the collinear limit $\theta_{i j}^{2} \rightarrow 0$ and in the soft limits $z \rightarrow 0$ and $(1-z) \rightarrow 0$. Of the two, $k_{\mathrm{T}}^{2}$ gives more emphasis to $z(1-z)$, so that virtuality ordering is intermediate between $k_{\mathrm{T}}$ ordering and angular ordering, which gives no emphasis at all to $z(1-z)$ and remains finite when a gluon becomes soft.

SHERPA and the current version of PYTHIA use versions of $k_{\mathrm{T}}^{2}$ as the ordering variable. We note that the previous version of PYтнIA is often known as "virtuality ordered PythiA," while the new one is often known as " $k_{\mathrm{T}}$ ordered PyтніA." However, in our opinion, the important difference between the new and old versions is not the choice of ordering variable but rather the inclusion in the new version of quantum interference effects in the final state shower, as in eq. (2.5).

\subsection{Definitions of transverse momentum}

To understand $k_{\mathrm{T}}$ for the splitting of a mother parton into partons $i$ and $j$, we use nullplane components of vectors, $v=\left(v^{+}, v^{-}, \boldsymbol{v}\right)$. Here $v^{ \pm}=\left(v^{0} \pm v^{3}\right) / \sqrt{2}$ and we use boldface to indicate vectors in the transverse plane. Then $v^{2}=2 v^{+} v^{-}-\boldsymbol{v}^{2}$.

We define the plus and minus components of vectors using lightlike basis vectors $u_{\mathrm{J}}$ and $n_{\mathrm{J}}$, where $u_{\mathrm{J}}$ represents the direction of a jet containing partons $i$ and $j$. Thus $u_{\mathrm{J}}$ is either approximately or exactly aligned with $p_{i}+p_{j}$. We define $n_{\mathrm{J}}$ to be the lightlike vector in the plane of $u_{\mathrm{J}}$ and the fixed vector $Q_{0}$ equal to the total momentum of the final state partons created by the hard process that initiates the shower. The idea is that $Q_{0}$ is fixed for a whole event, $\vec{Q}_{0}=0$ in the c.m. frame of the partonic scattering. We normalize the basis vectors to $u_{\mathrm{J}} \cdot Q_{0}=n_{\mathrm{J}} \cdot Q_{0}=\sqrt{Q_{0}^{2} / 2}$. Then also $u_{\mathrm{J}} \cdot n_{\mathrm{J}}=1$. Using these basis vectors, we define for any vector $v, v^{+}=v \cdot n_{\mathrm{J}}$ and $v^{-}=v \cdot u_{\mathrm{J}}$. The time component of a vector is $v^{0}=\left(v^{+}+v^{-}\right) / \sqrt{2}=$ $v \cdot Q_{0} / \sqrt{Q_{0}^{2}}$. That is, in this frame, the fixed vector $Q_{0}$ has only a time component.

The components of the daughter parton momenta $p_{i}$ and $p_{j}$ have the form

$$
\begin{aligned}
p_{i} & =\left(p_{i}^{+}, \frac{\left(\left[p_{i}^{+} /\left(p_{i}^{+}+p_{j}^{+}\right)\right] \boldsymbol{p}_{\tilde{i j}}+\boldsymbol{k}\right)^{2}}{2 p_{i}^{+}}, \frac{p_{i}^{+}}{p_{i}^{+}+p_{j}^{+}} \boldsymbol{p}_{\tilde{i j}}+\boldsymbol{k}\right), \\
p_{j} & =\left(p_{j}^{+}, \frac{\left(\left[p_{j}^{+} /\left(p_{i}^{+}+p_{j}^{+}\right)\right] \boldsymbol{p}_{\tilde{i j}}-\boldsymbol{k}\right)^{2}}{2 p_{j}^{+}}, \frac{p_{j}^{+}}{p_{i}^{+}+p_{j}^{+}} \boldsymbol{p}_{\tilde{i j}}-\boldsymbol{k}\right) .
\end{aligned}
$$


Here $\boldsymbol{p}_{\widetilde{i j}}=\boldsymbol{p}_{i}+\boldsymbol{p}_{j}$ is the total transverse momentum of the daughter partons in our chosen frame. We could have made $\boldsymbol{p}_{\tilde{i j}}$ vanish by slightly adjusting the direction of $u_{\mathrm{J}}$. Independently of whether $\boldsymbol{p}_{\tilde{i j}}$ vanishes, if $\boldsymbol{k}=0$ then $p_{i}$ and $p_{j}$ are exactly collinear. Thus $\boldsymbol{k}$ measures how far from being collinear $p_{i}$ and $p_{j}$ are. Thus $\boldsymbol{k}$ is a measure of the transverse momentum in the splitting.

With these definitions, we find

$$
\left(p_{i}+p_{j}\right)^{2}=\frac{\left(p_{i}^{+}+p_{j}^{+}\right)^{2}}{p_{i}^{+} p_{j}^{+}} \boldsymbol{k}^{2} .
$$

Define momentum fractions by

$$
\begin{aligned}
z & =\frac{p_{i} \cdot n}{\left(p_{i}+p_{j}\right) \cdot n}, \\
1-z & =\frac{p_{j} \cdot n}{\left(p_{i}+p_{j}\right) \cdot n} .
\end{aligned}
$$

Then we can write $k_{\mathrm{T}}^{2} \equiv \boldsymbol{k}^{2}$ as

$$
k_{\mathrm{T}}^{2}=z(1-z)\left(p_{i}+p_{j}\right)^{2} .
$$

This illustrates the physical meaning of $k_{\mathrm{T}}^{2}$ and shows us that the definition is frame dependent. To define it, we need not only $p_{i}$ and $p_{j}$ but also the lightlike vector $n$ in eq. (2.10).

In our approximation (2.7) and in work with $k_{\mathrm{T}}^{2}$ in later sections of this paper, we are considering partons that are part of a jet. All of these partons are moving in almost the same direction. We take $n$ to be a lightlike vector in the opposite direction to the jet of interest, as viewed in the rest frame of the hard interaction that initiates the shower. This definition of $k_{\mathrm{T}}^{2}$ is essentially the same as that used in the $k_{\mathrm{T}}$ jet algorithm for hadron collisions $[21,22]$.

However, this is not the definition of $n$ that is most often used in $k_{\mathrm{T}}$-ordered parton shower algorithms. The modern versions of these algorithms account for quantum interference, as in eq. (2.5). Then for splitting of a parton $l \rightarrow i+j$ there is another parton $k$ that is said to form a dipole with $l$. It is useful $[23,24]$ to describe this using at least some of the dipole emission formalism of Catani and Seymour [25]. This formalism is based on the momenta $p_{i}, p_{j}$, and $p_{k}$. Since $p_{k}$ is a lightlike vector (in the case of massless partons), it is convenient to take $n=p_{k}$. That is the definition in the default version of SHERPA [13] and also in the dipole showers defined in refs. [26, 27]. In the current version of PYTHIA, the definition is a little different: $n=p_{i}+p_{j}+p_{k}$. This is a timelike vector, not a lightlike vector. However, if $p_{i}$ and $p_{j}$ are nearly collinear, we have $p_{i} \cdot n \approx p_{i} \cdot p_{k}$ and $p_{j} \cdot n \approx p_{j} \cdot p_{k}$, so the difference between the definitions is small. The PYTHIA authors argue [28] that their definition is superior in the case that $p_{i}$ or $p_{j}$ becomes collinear with $p_{k}$.

Note that, as a shower develops, it is common for parton $k$ to be part of the same jet as the mother parton $l$ of partons $i$ and $j$. That is, $p_{k}$ is close to being collinear with the direction of the jet. In this case, $p_{i} \cdot n$ and $p_{j} \cdot n$ are small and $k_{\mathrm{T}}^{2}$ defined with $n=p_{k}$ is 
very different from $k_{\mathrm{T}}^{2}$ based on $n$ defined using the rest frame of the hard interaction, so that $p_{i} \cdot n$ and $p_{j} \cdot n$ are large. ${ }^{3}$

In summary, $k_{\mathrm{T}}^{2}$ defined with $n=p_{k}$ or $n=p_{i}+p_{j}+p_{k}$ is a good measure of hardness of a splitting in the sense that it vanishes in all limits in which $\left(p_{i}+p_{j}\right)^{2} \rightarrow 0$. However, it is not closely related to the $k_{\mathrm{T}}^{2}$ of a splitting as viewed in the overall rest frame of the event.

A very recent paper [29], which appeared after the present paper was submitted, examines the practical effect on some selected observables of choices of shower organization, including the standard choices of shower ordering variable outlined above.

\subsection{Summing large logarithms}

Parton shower event generators have the potential to sum large logarithms that appear order by order in perturbation theory. The choice of shower ordering variable can possibly have an impact on whether such logarithms are summed correctly.

One example of the summation of logarithms concerns the probability to find a quark with a given energy $E_{q}$ in a parton shower treatment of electron-positron annihilation. This distribution evolves as the evolution variable of the shower changes. The shower evolution sums logarithms of $V^{2} / s$. The distribution of $z=2 E_{q} / \sqrt{s}$ should evolve according to the DGLAP [30-32] evolution equation. A paper of Dokshitzer and Marchesini [33] raised issues about whether a dipole based shower using virtuality or $k_{\mathrm{T}}$ ordering could give DGLAP evolution, which was known to hold in an angle ordered shower. An analytical analysis of the shower evolution equations showed that the dipole showers with virtuality or $k_{\mathrm{T}}$ ordering do indeed produce DGLAP evolution of the quark energy distribution [34]. A separate analysis, including numerical comparisons, reached the same conclusions [35].

Another example of the summation of logarithms concerns the transverse momentum distribution of Z-bosons produced in hadron-hadron collisions. Here one hopes to sum logarithms of $P_{\mathrm{T}}^{2} / M_{\mathrm{Z}}^{2}$ and there are two logarithms per power of $\alpha_{\mathrm{s}}$ instead of just one in the DGLAP case. Again, there is a standard analytical result in QCD [36]. One can wonder whether a dipole based parton shower gets this summation right. One can also note that the natural shower evolution variable for summing logarithms of $P_{\mathrm{T}}^{2} / M_{\mathrm{Z}}^{2}$ would seem to be the $k_{\mathrm{T}}^{2}$ in each splitting. It is then an obvious question whether a virtuality ordered shower can get the right answer. An analytical analysis of the shower evolution equations showed that the dipole showers with virtuality or $k_{\mathrm{T}}$ ordering do indeed produce the correct summation of logarithms of $P_{\mathrm{T}}^{2} / M_{\mathrm{Z}}^{2}$ [37].

We should express a word of caution. It is true that in some cases parton shower algorithms can sum leading logarithms or next-to-leading logarithms (with a suitable definition of what "leading" and "next-to-leading" mean). However, it seems unlikely that this works in all cases. Before analyzing this question for a given shower algorithm, one needs to specify what observable one is looking at and what logarithms one hopes to sum. The verdict

\footnotetext{
${ }^{3}$ This same issue affects the recoil momentum that must be supplied to allow an on-shell mother parton to split to two on-shell daughters. In the strict Catani-Seymour construction, this momentum comes from parton $k$. This strategy becomes very sensitive to $p_{k}$ when $p_{k}$ is close to being collinear with $p_{i}$ and $p_{j}$. It is partly to avoid this problem that DEDUCTOR uses a global recoil strategy instead of the Catani-Seymour strategy.
} 
will depend on the observable. To take a simple example, one can define jets in electronpositron annihilation using the original Jade algorithm, based on sequentially combining protojets based on the invariant mass of the jets to be combined, $y_{i j}=\left(p_{i}+p_{j}\right)^{2} / s$. (For some jet algorithm definitions, see [38]). If we stop combining protojets at a fixed value $y_{\text {cut }}$ of $y_{i j}$, the perturbative expression for the fraction of two jets contains logarithms of $y_{\text {cut }}$. Although the Jade jet algorithm is infrared safe, it is usually regarded as unsuitable for use because the logarithms of $y_{\text {cut }}$ do not organize themselves in a nice way. It is not known how to sum these logarithms. We thus do not not know whether any current shower algorithms correctly sum these logarithms.

\section{Definition of ordering variable in Deductor}

A parton shower event generator for hadron-hadron scattering generates final states starting with some hard process. The shower includes both final state splittings and initial state splittings from the incoming partons. For each event, there is a shower history, $H$, as in figure 1. The event generator generates a given shower history with a probability $P_{H}$.

Now, the shower history has the appearance of a Feynman diagram, with its corresponding amplitude $\mathcal{M}_{H}$. In a parton shower, we wish to describe the propagation of partons over larger and larger distances as the shower progresses. We use a physical gauge for evaluating $\mathcal{M}_{H}$, so that only transversely polarized gluons can propagate over large distances. One might imagine that the probability $P_{H}$ is approximately proportional to the corresponding $\left|\mathcal{M}_{H}\right|^{2}, P_{H} \approx \mathcal{N}\left|\mathcal{M}_{H}\right|^{2}$. (The normalization factor $\mathcal{N}$ need not concern us here.) That can't be quite right because the event generator needs to account for interference graphs, in which a soft gluon is emitted from one parton in $\mathcal{M}_{H}$ and from a different parton in an amplitude $\mathcal{M}_{H^{\prime}}^{*}$. For that reason, we really need to discuss amplitudes $\mathcal{M}_{H}$. We will, in fact, discuss amplitudes, but let us ignore this issue for a first orientation. Then it is possible, but not necessary, that the $P_{H}$ is an approximation to $\mathcal{N}\left|\mathcal{M}_{H}\right|^{2}$ for the corresponding Feynman diagram. It is not necessary that $P_{H} \approx \mathcal{N}\left|\mathcal{M}_{H}\right|^{2}$ because all that one really needs is that $\sum_{H} P_{H}$ approximates $\mathcal{N} \sum_{H}\left|\mathcal{M}_{H}\right|^{2}$.

Nevertheless, we believe that it is desirable that $P_{H}$ approximates $\mathcal{N}\left|\mathcal{M}_{H}\right|^{2}$ (after accounting for interference graphs) and we used that idea as a design goal in constructing the parton shower algorithm used in DEDUCTOR. Maintaining this diagram by diagram correspondence constrains the choice of the ordering variable for shower evolution. Our purpose in this paper is to study this constraint and explain why we made the choice of shower time used in Deductor.

\subsection{Shower time for final state splittings}

In order to see why a correspondence between shower splitting probabilities and Feynman diagrams constrains the choice of shower time, it is easiest to start with final state splittings. Suppose that, in a shower history $H$ of interest, a final state parton 0 splits into partons 1 and 2, each of which splits further. This continues through some (finite) number of splittings. 
In the Feynman diagram corresponding to history $H$, the momenta of the mother parton and of the two daughters are related by $p_{0}=p_{1}+p_{2}$. Let the partons have masses $m_{i}, i=0,1,2$. We define corresponding virtualities $v_{i}^{2}$ by $v_{i}^{2}=p_{i}^{2}-m_{i}^{2}$. With final state splittings, we have $v_{i}^{2} \geq 0$. In our Feynman diagram, the propagator for the mother parton has a denominator $1 / v_{0}^{2}$. The daughter partons have propagators with denominators $1 / v_{1}^{2}$ and $1 / v_{2}^{2}$.

With the idea that the shower evolves from hard interactions to softer interactions, the picture for the Feynman diagram is that the splitting $0 \rightarrow 1+2$ should be relatively hard compared to the subsequent splittings of the daughter partons 1 and 2. In particular, we ought to be able to set $v_{1}^{2}$ and $v_{2}^{2}$ to 0 when calculating the propagator denominator $1 /\left[\left(p_{1}+\right.\right.$ $\left.\left.p_{2}\right)^{2}-m_{0}^{2}\right]=1 / v_{0}^{2}$ that controls the probability of the splitting $0 \rightarrow 1+2 .{ }^{4}$ This statement is of some practical significance. When the shower algorithm generates the splitting $0 \rightarrow 1+2$, the splittings of partons 1 and 2 have not yet been generated. Thus $v_{1}^{2}$ and $v_{2}^{2}$ are unknown. Knowing only that the daughter virtualities should be small, we set them to zero.

This qualitative argument suggests a design feature for a parton shower. The definition of the shower ordering variable should be such that one can neglect $v_{1}^{2}$ and $v_{2}^{2}$ when calculating $v_{0}^{2}$.

We now need to make more precise the proposition that one should be able to "neglect $v_{1}^{2}$ and $v_{2}^{2}$ when calculating $v_{0}^{2}$." We make use of the idea that parton showers are about the development of jets: groups of particles with large momenta approximately collinear to a certain direction, defined by a lightlike vector $u_{\mathrm{J}}$ as in section 2.4 . We follow our notation in earlier work and in section 2.4 by letting $Q_{0}$ denote the total momentum of the final state partons created by the hard process that initiates the shower. The subscript " 0 " here is not to be confused with the label " 0 " in our labeling of partons $0 \rightarrow 1+2$. The idea is that $Q_{0}$ is fixed for a whole event. We define components of vectors using a reference frame in which $Q_{0}$ has only a time component. This leaves the choice of $z$-axis free. We align the $z$-axis of our reference frame along the direction of the jet of interest. This amounts to defining a second lightlike basis vector $n_{\mathrm{J}}$ in the $u_{\mathrm{J}}-Q_{0}$ plane, as in section 2.4.

We suppose that the mother parton is part of the jet of interest and we describe its momentum $p_{0}$ using $(+,-, \perp)$ components in a reference frame defined with the basis vectors $u_{\mathrm{J}}$ and $n_{\mathrm{J}}$ as in section 2.4. In this frame, partons in the jet have momenta with large plus components and small minus components: $p^{+} \gg|\boldsymbol{p}| \gg p^{-}$, where the transverse components are denoted by a boldface symbol, $\boldsymbol{p}$. The components of $p_{0}$ are

$$
p_{0}=\left(P, \frac{\boldsymbol{p}_{0}^{2}+m_{0}^{2}+v_{0}^{2}}{2 P}, \boldsymbol{p}_{0}\right) .
$$

Here $m_{0}$ is the mass of the mother parton, $\boldsymbol{p}_{0}$ is its momentum transverse to the jet direction, and $v_{0}^{2}$ is its virtuality. With our choice of reference frame, $P$ is large: $P^{2} \gg \boldsymbol{p}_{0}^{2}$, $P^{2} \gg m_{0}^{2}, P^{2} \gg v_{0}^{2}$. We could have chosen the reference frame so that $\boldsymbol{p}_{0}=0$, but we

\footnotetext{
${ }^{4}$ Large logarithms are a feature of parton showers. In this situation we generate a large logarithm from an integration $\int d v_{0}^{2} / v_{0}^{2}$, integrated over a wide range of $v_{0}^{2}$. A second power of the logarithm comes from integrating over the momentum fraction $z$ in the splitting.
} 
leave the choice general. Parton 0 splits into two partons with momenta

$$
\begin{aligned}
& p_{1}=\left(z P, \frac{\boldsymbol{p}_{1}^{2}+m_{1}^{2}+v_{1}^{2}}{2 z P}, \boldsymbol{p}_{1}\right), \\
& p_{2}=\left((1-z) P, \frac{\boldsymbol{p}_{2}^{2}+m_{2}^{2}+v_{2}^{2}}{2(1-z) P}, \boldsymbol{p}_{2}\right) .
\end{aligned}
$$

Here $z$ and $(1-z)$ are the momentum fractions carried by the daughter partons. Their transverse momenta are related by

$$
\boldsymbol{p}_{1}+\boldsymbol{p}_{2}=\boldsymbol{p}_{0} .
$$

Partons 1 and 2 are off shell with virtualities $v_{1}^{2}$ and $v_{2}^{2}$, respectively. Setting $p_{0}^{-}=p_{1}^{-}+p_{2}^{-}$ and solving for $v_{0}$, we find

$$
v_{0}^{2}=\frac{\left((1-z) \boldsymbol{p}_{1}-z \boldsymbol{p}_{2}\right)^{2}}{z(1-z)}+\frac{m_{1}^{2}}{z}+\frac{m_{2}^{2}}{1-z}-m_{0}^{2}+\frac{v_{1}^{2}}{z}+\frac{v_{2}^{2}}{(1-z)} .
$$

If we neglect $v_{1}^{2}$ and $v_{2}^{2}$ when calculating $v_{0}^{2}$, we obtain $\tilde{v}_{0}^{2}$, where

$$
\tilde{v}_{0}^{2}=\frac{\left((1-z) \boldsymbol{p}_{1}-z \boldsymbol{p}_{2}\right)^{2}}{z(1-z)}+\frac{m_{1}^{2}}{z}+\frac{m_{2}^{2}}{1-z}-m_{0}^{2} .
$$

The approximation $v_{0}^{2} \approx \tilde{v}_{0}^{2}$ is a good approximation provided

$$
\frac{v_{1}^{2}}{z} \ll v_{0}^{2}, \quad \frac{v_{2}^{2}}{1-z} \ll v_{0}^{2} .
$$

That is

$$
\frac{v_{1}^{2}}{2 p_{1} \cdot n} \ll \frac{v_{0}^{2}}{2 p_{0} \cdot n}, \quad \frac{v_{2}^{2}}{2 p_{2} \cdot n} \ll \frac{v_{0}^{2}}{2 p_{0} \cdot n} .
$$

where $n$ is the lightlike vector $n=(0,1, \mathbf{0})$.

We want eq. (3.7) to hold whenever the splittings of the two daughter partons come after the splitting $0 \rightarrow 1+2$. To guarantee that, we want to arrange the definition of shower time such that the splitting of parton 1 comes after the splitting of parton 0 when the first of conditions (3.7) holds and such that the splitting of parton 2 comes after the splitting of parton 0 when second of conditions (3.7) holds.

These ordering conditions are a simple restatement of the requirement that $v_{0}^{2} \approx \tilde{v}_{0}^{2}$. But what is $\tilde{v}_{0}^{2}$ ? It is the limit of $v_{0}^{2}$ as $v_{1}^{2} \rightarrow 0$ and $v_{2}^{2} \rightarrow 0$ with fixed $\boldsymbol{p}_{1}, \boldsymbol{p}_{2}$, and $z$. This was a deliberate choice. It was motivated as follows. We define $\tilde{v}_{0}^{2}=\left(\tilde{p}_{1}+\tilde{p}_{2}\right)^{2}-m_{0}^{2}$, where $\tilde{p}_{1}$ and $\tilde{p}_{2}$ are on-shell approximations to $p_{1}$ and $p_{2}: \tilde{p}_{i}^{2}=m_{i}^{2}$ for $i=1,2$. We want the components of $\delta p_{i}=p_{i}-\tilde{p}_{i}$ to be small. Since $v_{i}^{2}=\left(\tilde{p}_{i}^{2}+\delta p_{i}\right)^{2}-m_{i}^{2}=2 \tilde{p}_{i} \cdot \delta p_{i}+\left(\delta p_{i}\right)^{2}$, we have, neglecting $\left(\delta p_{i}\right)^{2}$,

$$
v_{i}^{2} / 2 \approx \tilde{p}_{i}^{+} \delta p_{i}^{-}-\tilde{\boldsymbol{p}}_{i} \cdot \delta \boldsymbol{p}_{i}+\tilde{p}_{i}^{-} \delta p_{i}^{+} .
$$

Now, $\tilde{p}_{i}^{+} \gg\left|\tilde{\boldsymbol{p}}_{i}\right| \gg \tilde{p}_{i}^{-}$. Thus we can make the components of $\delta p_{i}$ small by choosing

$$
\delta p^{-}=\frac{v_{i}^{2}}{2 \tilde{p}_{i}^{+}}
$$


and letting $\delta \boldsymbol{p}_{i}=0$ and $\delta p_{i}^{+}=0$. One could also let $\delta \boldsymbol{p}_{i}$ and $\delta p_{i}^{+}$be small, of a similar size to $\delta p^{-}$, but then $\delta p^{-}$is still determined approximately by eq. (3.9). Such a choice is equivalent to the simple choice that we make here. ${ }^{5}$

We need one more step in order to turn eq. (3.7) into a definition of shower time. Since the plus components of the parton momenta $p_{0}, p_{1}$ and $p_{2}$ in this frame are all much larger than their transverse and minus components, we have

$$
p_{i} \cdot n \approx p_{i} \cdot Q_{0} \sqrt{2 / Q_{0}^{2}}
$$

Then we can write the ordering conditions (approximately) as

$$
\frac{v_{1}^{2}}{2 p_{1} \cdot Q_{0}} \ll \frac{v_{0}^{2}}{2 p_{0} \cdot Q_{0}}, \quad \frac{v_{2}^{2}}{2 p_{2} \cdot Q_{0}} \ll \frac{v_{0}^{2}}{2 p_{0} \cdot Q_{0}} .
$$

This leads us to the definition of shower time for a final state splitting. For the splitting of parton $i$, we take

$$
e^{-t_{i}}=\frac{\Lambda_{i}^{2}}{Q_{0}^{2}}
$$

where we define the ordering variable $\Lambda$ by

$$
\Lambda_{i}^{2}=\frac{p_{i}^{2}-m_{i}^{2}}{2 p_{i} \cdot Q_{0}} Q_{0}^{2}
$$

We emphasize again that $Q_{0}$ is a fixed vector that defines the hard scattering c.m. system.

With this definition, the splittings of partons 1 and 2 come after the splitting of parton 0 when the conditions (3.11) hold. Of course, within a parton shower we calculate $p_{i}^{2}$ as the square of the sum of the momenta of the daughter partons to parton $i$, using the approximation that the daughter partons are on shell. Additionally, at each splitting a small amount of momentum needs to be taken from the existing final state partons in the event, as described in section 4.1 of ref. [3]. Thus, there is an ambiguity in how to calculate $p_{i} \cdot Q_{0}$. We calculate $p_{i} \cdot Q_{0}$ defining $p_{i}$ to be the momentum of the parton $i$ as it existed when the parton was created, before it splits.

In the shower algorithm, we take "after" to mean $t_{1}>t_{0}$ and $t_{2}>t_{0}$. This replaces $\ll$ in eq. (3.11) by simply $<$. This does not treat the regions $t_{1} \approx t_{0}$ and $t_{2} \approx t_{0}$ very exactly. We recognize this as a shortcoming of a leading order shower that can be fixed if we move on to a next-to-leading order shower.

It may be helpful to consider an example. We use massless partons and let the fixed vector $Q_{0}$ be $Q_{0}^{0}=500 \mathrm{GeV}, Q_{0}^{1}=Q_{0}^{2}=Q_{0}^{3}=0$ in the reference frame that we use. Consider the splitting of a mother parton with momentum $p_{0}$ to a daughter parton with momentum $p_{1}$ and a second daughter parton. Let the energy of the mother parton before the splitting be $p_{0}^{0}=100 \mathrm{GeV}$. Let $p_{1}^{0}=E$ be the energy of daughter parton 1 and let

\footnotetext{
${ }^{5}$ For instance, when the partons are part of a high energy jet, if we define $\tilde{v}_{0}^{2}$ by taking the limit $v_{1}^{2} \rightarrow 0$ and $v_{2}^{2} \rightarrow 0$ with the three-momenta of the partons held constant, adjusting the daughter parton energies a little to put them on-shell, we obtain the same final definition of shower time.
} 

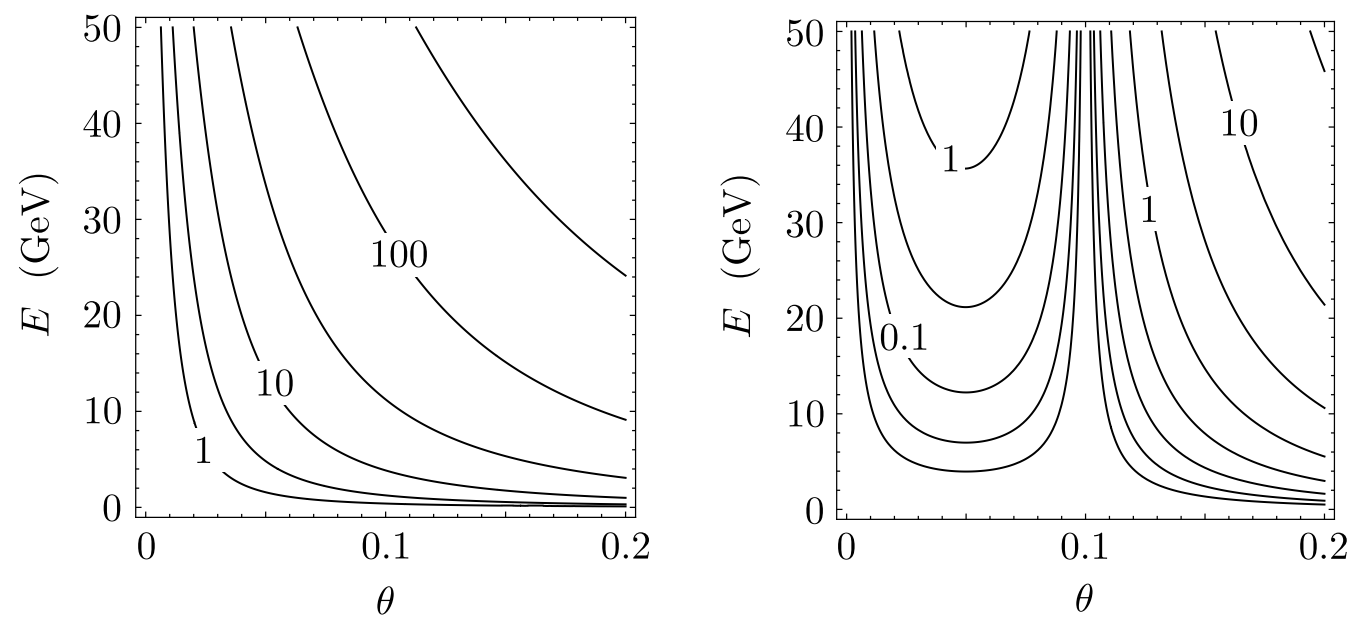

Figure 3. Example of contours of $\Lambda^{2}$ and $k_{\mathrm{T}}^{2}$ (in $\mathrm{GeV}^{2}$ units) as functions of daughter parton energy $E$ and angle $\theta$ with respect to the mother parton. The left hand plot shows $\Lambda^{2}$ and the right hand plot shows $k_{\mathrm{T}}^{2}$. We take the helper parton to make an angle 0.1 with respect to the mother parton.

$\vec{p}_{1}$ make an angle $\theta$ with respect to $\vec{p}_{0} \cdot{ }^{6}$ In a partitioned dipole shower like Deductor, there is a helper parton $k$ that, together with the mother parton, forms a color dipole. We take $\vec{p}_{k}$ to make an angle $\theta_{k}=0.1$ with respect $\vec{p}_{0}$ and we take $\vec{p}_{1}$ to lie in the plane of $\vec{p}_{0}$ and $\vec{p}_{k}: \vec{p}_{1}=\alpha \vec{p}_{0}+\beta \vec{p}_{k}$ with positive $\beta$. This allows $\vec{p}_{1}$ to be collinear with $\vec{p}_{k}$, but note that that the splitting function in Deductor is not singular when $\vec{p}_{1}=\beta \vec{p}_{k}$ : the $\vec{p}_{1} \| \vec{p}_{k}$ collinear singularity is part of the splitting function for the splitting of parton $k$.

In the left hand plot of figure 3, we exhibit contour lines of $\Lambda^{2}$ as a function of $E$ and $\theta$. Notice that $\Lambda^{2} \rightarrow 0$ as $E \rightarrow 0$ and also as $\theta \rightarrow 0$. In the right hand plot of figure 3 , we exhibit contour lines of $k_{\mathrm{T}}^{2}$, as defined in eqs. (2.10) and (2.11), with the choice $n=p_{k}$, which is commonly used for final state splittings in a $k_{\mathrm{T}}$-ordered parton shower. Notice that $k_{\mathrm{T}}^{2} \rightarrow 0$ as $E \rightarrow 0$ and also as $\theta \rightarrow 0$. Notice also that $k_{\mathrm{T}}^{2} \rightarrow 0$ as $\theta \rightarrow \theta_{k}$ so that $\vec{p}_{1} \rightarrow \beta \vec{p}_{k}$. This is a feature of the choice $n=p_{k}$ in the definition of $k_{\mathrm{T}}$.

\subsection{Shower time and physical time}

Let us look again at the shower time for a splitting $0 \rightarrow 1+2$. We use $(+,-, \perp)$ components as in eq. (3.1), defined using the basis vectors $u_{\mathrm{J}}$ and $n_{\mathrm{J}}$ from section 2.4. Using these momentum components, the shower time for the splitting of the mother parton 0 is

$$
e^{-t}=\frac{v_{0}^{2}}{2 p_{0} \cdot Q_{0}} \approx \sqrt{\frac{2}{Q_{0}^{2}}} \frac{v_{0}^{2}}{2 P}=\sqrt{\frac{2}{Q_{0}^{2}}}\left[p_{0}^{-}-\frac{p_{0}^{2}+m_{0}^{2}}{2 P}\right] .
$$

Here $\left(\boldsymbol{p}_{0}^{2}+m_{0}^{2}\right) /(2 P)$ is what the minus component of the momentum $p_{0}$ would be if the mother parton were on shell. When $p_{0}^{-}$does not equal this value, there is a deficit of minus

\footnotetext{
${ }^{6}$ In a parton shower, the mother parton was approximated as being on shell before the splitting. Once the splitting has been generated, we need to take a small amount of momentum from elsewhere in the event in order to conserve momentum at the splitting vertex. We use the momentum mapping of DeDUCTOR for this purpose. This choice affects the figure only slightly.
} 
momentum and the parton can only exist in this state for an interval in $x^{+}$given by the inverse of the minus-momentum deficit. That is, the interval in $x^{+}$between the vertex where the mother parton was created and where it splits can be estimated by

$$
\Delta x^{+} \sim\left[p_{0}^{-}-\frac{\boldsymbol{p}_{0}^{2}+m_{0}^{2}}{2 P}\right]^{-1} .
$$

The interval in space-time, $\Delta x^{\mu}$, between the two vertices is approximately in the plusdirection, so the corresponding time interval is $\Delta t=\Delta x^{0}=\left(\Delta x^{+}+\Delta x^{-}\right) \sqrt{2} \approx \Delta x^{+} / \sqrt{2}$. That is,

$$
\Delta t \sim \frac{1}{\sqrt{2}}\left[p_{0}^{-}-\frac{\boldsymbol{p}_{0}^{2}+m_{0}^{2}}{2 P}\right]^{-1} .
$$

This gives

$$
e^{-t} \sim \frac{1}{\sqrt{Q_{0}^{2}} \Delta t}
$$

or

$$
t \sim \log \left(\sqrt{Q_{0}^{2}} \Delta t\right)
$$

That is, the shower time at which a parton splits is an estimate of the logarithm of the coordinate time interval needed for the splitting, normalized by $\sqrt{Q_{0}^{2}}$ to make it dimensionless. Here the coordinate time interval is measured in a reference frame in which the fixed vector $Q_{0}$ has only a time component.

\subsection{Shower time for initial state splittings}

We now repeat the derivation of section 3.1, this time for initial state splittings in collisions of two hadrons $\mathrm{A}$ and $\mathrm{B}$. We approximate the hadron momenta $p_{\mathrm{A}}$ and $p_{\mathrm{B}}$ to be lightlike, with $2 p_{\mathrm{A}} \cdot p_{\mathrm{B}}=s$.

Our derivation will also use the the fixed vector $Q_{0}$ from section 2.4, equal to the total momentum of the final state partons just after the hard interaction. Here, there is a small subtlety that we should mention. In DeDUCTOR 1.0.0, we have chosen to keep the lab-frame components $Q_{0}^{\mu}$ of $Q_{0}$ fixed throughout the shower. Then $Q_{0}$ is exactly in the plane of $p_{\mathrm{A}}$ and $p_{\mathrm{B}}$. However, at each shower splitting, DEDUCTOR applies a Lorentz transformation to the current final state parton momenta in order to conserve momentum and, in an initial state splitting, allow the new initial state parton to have zero transverse momentum. This is described in ref. [3] for final state splittings and in section 5.3 and appendix A for initial state splittings. If one regards this as a change of reference frame rather than as an active transformation of momenta, then one could apply this Lorentz transformation also to $Q_{0}$. With that choice, $Q_{0}$ is not exactly in the plane of $p_{\mathrm{A}}$ and $p_{\mathrm{B}}$, although the part of $Q_{0}$ transverse to $p_{\mathrm{A}}$ and $p_{\mathrm{B}}$ will typically be small. In order to keep our description general, in this section we allow $Q_{0}$ to have a relatively small part transverse to $p_{\mathrm{A}}$ and $p_{\mathrm{B}}$. Additionally, we allow initial state parton momenta to have relatively small transverse parts.

To describe an initial state splitting, we will use $(+,-, \perp)$ components of vectors and choose the axes so that $p_{\mathrm{A}}$ has only a plus component and $p_{\mathrm{B}}$ has only a minus component. This specification does not fix the ratio of plus components of vectors to their minus 
components. For that, we choose the reference frame so that $Q_{0}^{+}=Q_{0}^{-}$. As discussed above, we allow for the possibility that $Q_{0}$ has transverse part $\boldsymbol{Q}_{0}$, with $\boldsymbol{Q}_{0}^{2} \ll Q_{0}^{2}$.

In our chosen frame, initial state partons from hadron A have large plus components, small transverse components, and very small minus components, while initial state partons from hadron B have large minus components, small transverse components, and very small plus components.

We start with an initial state parton from hadron A with momentum

$$
p_{0}=\left(P, \frac{\boldsymbol{p}_{0}^{2}+m_{0}^{2}+v_{0}^{2}}{2 P}, \boldsymbol{p}_{0}\right) .
$$

Here $v_{0}^{2}=p_{0}^{2}-m_{0}^{2}$, the virtuality of the initial state parton, is negative. This parton splits (in backward evolution) into two partons, 1 and 2. Parton 1 is the new initial state parton, while parton 2 is radiated. Thus

$$
p_{0}=p_{1}-p_{2} .
$$

We define

$$
\begin{aligned}
& p_{1}=\left(\frac{1}{z} P, z \frac{\boldsymbol{p}_{1}^{2}+m_{1}^{2}+v_{1}^{2}}{2 P}, \boldsymbol{p}_{1}\right) \\
& p_{2}=\left(\frac{1-z}{z} P, \frac{z}{1-z} \frac{\boldsymbol{p}_{2}^{2}+m_{2}^{2}+v_{2}^{2}}{2 P}, \boldsymbol{p}_{2}\right) .
\end{aligned}
$$

Here $v_{1}^{2} \leq 0$ and $v_{2}^{2} \geq 0$.

We can easily find the virtuality $v_{0}^{2}$ by comparing the minus momentum of parton 0 to the difference of the minus momenta of partons 1 and 2 :

$$
v_{0}^{2}=z v_{1}^{2}-\frac{z}{1-z} v_{2}^{2}-\frac{1}{1-z}\left(\boldsymbol{p}_{2}-(1-z) \boldsymbol{p}_{1}\right)^{2}+z m_{1}^{2}-\frac{z}{1-z} m_{2}^{2}-m_{0}^{2} .
$$

We note that we can approximate $v_{0}^{2}$ by its value for on-shell daughter partons,

$$
v_{0}^{2} \approx-\frac{1}{1-z}\left(\boldsymbol{p}_{2}-(1-z) \boldsymbol{p}_{1}\right)^{2}+z m_{1}^{2}-\frac{z}{1-z} m_{2}^{2}-m_{0}^{2},
$$

provided that

$$
z\left|v_{1}^{2}\right| \ll\left|v_{0}^{2}\right|, \quad \frac{z}{1-z} v_{2}^{2} \ll\left|v_{0}^{2}\right| .
$$

That is

$$
\frac{\left|v_{1}^{2}\right|}{2 p_{1} \cdot n} \ll \frac{\left|v_{0}^{2}\right|}{2 p_{0} \cdot n}
$$

and

$$
\frac{v_{2}^{2}}{2 p_{2} \cdot n} \ll \frac{\left|v_{0}^{2}\right|}{2 p_{0} \cdot n},
$$

where $n$ is the lightlike vector $n=(0,1, \mathbf{0})$ in the direction of $p_{\mathrm{B}}$.

For the parton "2" that was radiated into the final state, since $p_{2}^{+} \gg p_{2}^{\perp} \gg p_{2}^{-}$, we have $2 p_{2} \cdot n \approx 2 p_{2} \cdot Q_{0} \sqrt{2 / Q_{0}^{2}}$. For the initial state partons "0" and "1," this same 
approximation is valid. However, later formulas are nicer if we stick with $p_{0} \cdot n$ and $p_{1} \cdot n$ using the lightlike vector $n$. A useful notation for this is provided by writing

$$
\begin{aligned}
& p_{0} \cdot n \approx \eta_{0} p_{\mathrm{A}} \cdot Q_{0} \sqrt{2 / Q_{0}^{2}}, \\
& p_{1} \cdot n \approx \eta_{1} p_{\mathrm{A}} \cdot Q_{0} \sqrt{2 / Q_{0}^{2}},
\end{aligned}
$$

where the momentum fractions $\eta_{0}$ and $\eta_{1}$ are defined by

$$
\begin{aligned}
\eta_{0} & =2 p_{0} \cdot p_{\mathrm{B}} / s, \\
\eta_{1} & =2 p_{1} \cdot p_{\mathrm{B}} / s .
\end{aligned}
$$

With this notation, we can write the conditions for neglecting $v_{1}^{2}$ and $v_{2}^{2}$ as

$$
\frac{\left|v_{1}^{2}\right|}{2 \eta_{1} p_{\mathrm{A}} \cdot Q_{0}} \ll \frac{\left|v_{0}^{2}\right|}{2 \eta_{0} p_{\mathrm{A}} \cdot Q_{0}}, \quad \frac{v_{2}^{2}}{2 p_{2} \cdot Q_{0}} \ll \frac{\left|v_{0}^{2}\right|}{2 \eta_{0} p_{\mathrm{A}} \cdot Q_{0}} .
$$

This leads us to define the shower time for an initial state splitting that is analogous to eq. (3.13) for a final state splitting,

$$
\begin{aligned}
& e^{-t_{i}}=\frac{\Lambda_{i}^{2}}{Q_{0}^{2}}=\frac{p_{i}^{2}-m_{i}^{2}}{2 p_{i} \cdot Q_{0}} \quad \text { final state parton }, \\
& e^{-t_{i}}=\frac{\Lambda_{i}^{2}}{Q_{0}^{2}}=\frac{\left|p_{i}^{2}-m_{i}^{2}\right|}{2 \eta_{i} p_{\mathrm{A}} \cdot Q_{0}} \quad \text { initial state parton, }
\end{aligned}
$$

with an analogous equation for an initial state parton from hadron B. Because at each splitting a small amount of momentum needs to be taken from the existing final state partons in the event, as described in section 5.3 below, there is an ambiguity in how exactly to calculate $\eta_{i}$. We define $\eta_{i}$ to be the momentum fraction of the initial state parton as it existed when the parton was created, before it splits.

\subsection{Interference graphs}

A parton with label $l$ can emit a gluon, giving an amplitude $\mathcal{M}_{l}$ (in a physical gauge). We have, so far, analyzed the choice of ordering for squared amplitudes, $\left|\mathcal{M}_{l}\right|^{2}$. However, we need to account for quantum interference. A parton with label $k$, moving in a different direction, can emit the gluon, giving an amplitude $\mathcal{M}_{k}$. When the gluon is soft and its direction is not highly collinear with either parton $l$ or parton $k$, both processes are important and should be included in a parton shower. Thus we need to account for interference contributions $\mathcal{M}_{l} \mathcal{M}_{k}^{*}$ and $\mathcal{M}_{k} \mathcal{M}_{l}^{*}$, as illustrated in figure 4

The parton shower algorithm of $[1,3,4]$ takes such interference contributions into account, at least approximately. To understand how this works, consider emission of a gluon with momentum $\hat{p}_{\mathrm{g}}$ from parton $l$ with interference from emission from parton $k$. The momenta of the partons $l$ and $k$ are $p_{l}$ and $p_{k}$ before the emission and $\hat{p}_{l}$ and $\hat{p}_{k}$ after the emission. The probability for this emission in the limit that the gluon is soft, $\hat{p}_{\mathrm{g}} \rightarrow 0$, is proportional to the dipole splitting function

$$
\bar{w}_{l k}^{\text {dipole }}=4 \pi \alpha_{\mathrm{s}} \frac{-\left(\hat{p}_{\mathrm{g}} \cdot \hat{p}_{l} \hat{p}_{k}-\hat{p}_{\mathrm{g}} \cdot \hat{p}_{k} \hat{p}_{l}\right)^{2}}{\left(\hat{p}_{\mathrm{g}} \cdot \hat{p}_{k} \hat{p}_{\mathrm{g}} \cdot \hat{p}_{l}\right)^{2}} .
$$




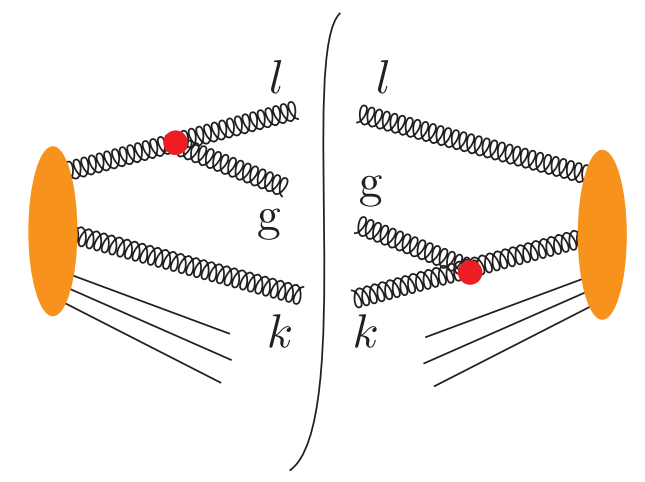

Figure 4. Emission of a soft gluon $\mathrm{g}$ from parton $l$ in $\mathcal{M}$ and from parton $k$ in $\mathcal{M}^{*}$.

This splitting function is described in more detail in sections (5.3) and (5.5) of ref. [6]. In eq. (3.31), partons $l$ and $k$ can have nonzero masses. The expression for $\bar{w}_{l k}^{\text {dipole }}$ is simpler in the massless limit, $\hat{p}_{l}^{2} \rightarrow 0$ and $\hat{p}_{k}^{2} \rightarrow 0$, where it becomes

$$
\bar{w}_{l k}^{\text {dipole }} \rightarrow 4 \pi \alpha_{\mathrm{s}} \frac{2 \hat{p}_{k} \cdot \hat{p}_{l}}{\hat{p}_{\mathrm{g}} \cdot \hat{p}_{k} \hat{p}_{\mathrm{g}} \cdot \hat{p}_{l}} .
$$

This splitting function multiplies an appropriate matrix $C_{l k}$ in color space, with $C_{l k}=C_{k l}$. Eq. (3.31) includes all four diagrams for emission from either parton $l$ or parton $k$ in the amplitude and the conjugate amplitude, calculated in the limit $\hat{p}_{\mathrm{g}}=\lambda P_{\mathrm{g}}$ with $\lambda \rightarrow 0$.

We introduce a partitioning function $A_{l k}^{\prime}$ with the properties that $A_{l k}^{\prime}>0$ and $A_{l k}^{\prime}+$ $A_{k l}^{\prime}=1$ as follows ${ }^{7}$

$$
\begin{aligned}
\frac{1}{2} \sum_{l} \sum_{k \neq l} \bar{w}_{l k}^{\text {dipole }} C_{l k} & =\frac{1}{2} \sum_{l} \sum_{k \neq l}\left[A_{l k}^{\prime}+A_{k l}^{\prime}\right] \bar{w}_{l k}^{\text {dipole }} C_{l k} \\
& =\sum_{l} \sum_{k \neq l} A_{l k}^{\prime} \bar{w}_{l k}^{\text {dipole }} C_{l k} .
\end{aligned}
$$

We define $A_{l k}^{\prime}$ as in eq. (7.12) of ref. [5]:

$$
A_{l k}^{\prime}=\frac{\hat{p}_{\mathrm{g}} \cdot \hat{p}_{k} \hat{p}_{l} \cdot \hat{Q}}{\hat{p}_{\mathrm{g}} \cdot \hat{p}_{k} \hat{p}_{l} \cdot \hat{Q}+\hat{p}_{\mathrm{g}} \cdot \hat{p}_{l} \hat{p}_{k} \cdot \hat{Q}} .
$$

Here $\hat{Q}$ is the total momentum of the final state particles just after the splitting. We see that $A_{l k}^{\prime} \rightarrow 1$ and $A_{k l}^{\prime} \rightarrow 0$ when $\hat{p}_{\mathrm{g}}$ becomes collinear with $\hat{p}_{l}$. We treat the term $A_{l k}^{\prime} \bar{w}_{l k}^{\text {dipole }} C_{l k}$ as primarily describing the emission of the gluon from parton $l$, with parton $k$ playing a passive role as a spectator. For instance, there is a momentum mapping that takes a small amount of momentum from the rest of the partons in the event and delivers it to the partons involved in the splitting so that they can be on shell both before and after the splitting. We use the momentum mapping associated with $p_{l} \rightarrow \hat{p}_{l}+\hat{p}_{\mathrm{g}}$ and simply

\footnotetext{
${ }^{7}$ The color factors $C_{l k}$ are the factors in square brackets in eq. (5.7) of ref. [6]. Equation (3.33) appeared as eq. (5.8) of that paper, but there we inadvertently left out the color factors $C_{l k}$.
} 
ignore the small momentum transfer to parton $k$. More importantly for the topic of this paper, we define the shower time from the splitting $p_{l} \rightarrow \hat{p}_{l}+\hat{p}_{\mathrm{g}}$.

This seems rather crude. Can it be sensible? Note that the shower time associated with gluon emission from parton $l$ is $t=-\log \left(\Lambda_{l}^{2} / Q_{0}^{2}\right)$, where

$$
\Lambda_{l}^{2} \approx \frac{\left|\left(\hat{p}_{l} \pm \hat{p}_{\mathrm{g}}\right)^{2}-m_{l}^{2}\right|}{2\left(\hat{p}_{l} \pm \hat{p}_{\mathrm{g}}\right) \cdot Q_{0}} Q_{0}^{2}=\frac{2 \hat{p}_{l} \cdot \hat{p}_{\mathrm{g}}}{2\left(\hat{p}_{l} \pm \hat{p}_{\mathrm{g}}\right) \cdot Q_{0}} Q_{0}^{2} .
$$

Here the + sign is for a final state splitting while the - sign is for an initial state splitting. Of course, the same definition with $l \rightarrow k$ applies for $\Lambda_{k}^{2}$. For small $\hat{p}_{\mathrm{g}}$, this becomes

$$
\Lambda_{l}^{2} \approx \frac{\hat{p}_{l} \cdot \hat{p}_{\mathrm{g}}}{\hat{p}_{l} \cdot Q_{0}} Q_{0}^{2} .
$$

Compare this to the same function using $\hat{Q}$ in place of $Q_{0}$ :

$$
\widetilde{\Lambda}_{l}^{2} \approx \frac{\hat{p}_{l} \cdot \hat{p}_{\mathrm{g}}}{\hat{p}_{l} \cdot \hat{Q}} \hat{Q}^{2} .
$$

We have

$$
\widetilde{\Lambda}_{l}^{2} \approx \alpha_{l} \Lambda_{l}^{2}
$$

where

Thus

$$
\alpha_{l}=\frac{\hat{Q}^{2}}{Q_{0}^{2}} \frac{\hat{p}_{l} \cdot Q_{0}}{\hat{p}_{l} \cdot \hat{Q}}
$$

$$
A_{l k}^{\prime} \approx \frac{\alpha_{k} \Lambda_{k}^{2}}{\alpha_{l} \Lambda_{l}^{2}+\alpha_{k} \Lambda_{k}^{2}}
$$

Note that only the ratio of $\alpha_{l}$ to $\alpha_{k}$, not their individual values, matters in $A_{l k}^{\prime}$. Furthermore, $\alpha_{l} / \alpha_{k}$ is typically neither much larger than 1 nor much smaller than 1 .

What happens? We can consider three cases.

First, one can have $\Lambda_{l}^{2} \ll \Lambda_{k}^{2}$. Then $A_{l k}^{\prime} \approx 1$ and $A_{k l}^{\prime} \approx 0$, so the splitting is treated almost entirely as gluon emission from parton $l$, for which $\Lambda_{l}^{2}$ defines the shower time. Thus $\Lambda_{l}^{2}$ must be smaller than the $\Lambda^{2}$ of the previous splitting in the shower. In this case, $\Lambda_{k}^{2}$ is much larger than $\Lambda_{l}^{2}$ and may be larger than the $\Lambda^{2}$ of the previous splitting, so that the approximations needed to neglect $\left(\hat{p}_{k} \pm \hat{p}_{\mathrm{g}}\right)^{2}-m_{k}^{2}$ in the previous splitting that produced parton $k$ may not be valid. However, in this case, if we examine the graphs that go into $\bar{w}_{l k}^{\text {dipole }}$, using a physical gauge $\hat{Q} \cdot A=0$, we find that the dominant graph is the one in which the soft gluon is emitted from parton $l$ both in the amplitude and in the conjugate amplitude. Graphs involving emission from parton $k$ are suppressed. Thus it does not matter if the approximations do not work well for emission from parton $k$.

Second, one can have $\Lambda_{k}^{2} \ll \Lambda_{l}^{2}$. This is the same as the previous case but with $l \leftrightarrow k$.

In the third case, $\Lambda_{k}^{2}$ and $\Lambda_{l}^{2}$ are of a similar size. In this case, we really do have substantial quantum interference in a physical gauge. The shower algorithm sometimes uses $\Lambda_{l}^{2}$ to define the shower time and sometimes uses $\Lambda_{k}^{2}$. However, it does not much matter which $\Lambda^{2}$ value is used because the two are of similar size.

One could, of course, use $Q_{0}$ in place of $\hat{Q}$ in the definition of $A_{l k}^{\prime}$. Then the argument given above would be simpler. Our only reason for not doing that is that the code for generating parton splittings is somewhat simpler with $A_{l k}^{\prime}$ defined using $\hat{Q}$. 


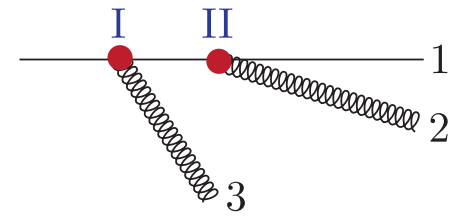

$A$

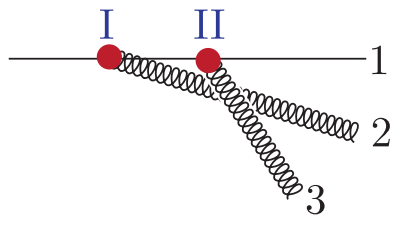

$B$

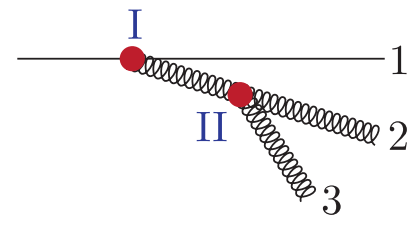

$C$

Figure 5. Three possible shower histories.

\section{Consequences for final state splittings}

In this section, we explore the consequences of the choice of ordering variable in final state splittings. For this purpose, we examine a simple example in which a final state quark splits into a quark, labeled 1, and two gluons, labeled 2 and 3. For simplicity, in this section we take the quark to be massless. There are three possible shower histories, illustrated in figure 5 . We examine whether the set of histories that are generated for a given $p_{1}, p_{2}$ and $p_{3}$ depends on the choice of the shower time variable.

We will consider using two shower ordering variables. We will find that in a certain region of $p_{1}, p_{2}$ and $p_{3}$, one ordering variable picks out history $\mathrm{A}$ while the other picks out histories $\mathrm{B}$ and $\mathrm{C}$. We will also find that the difference does not matter much because of color coherence: the probability calculated in a shower approximation according to history A equals approximately the sum of the probabilities calculated according to histories B and C.

\subsection{Kinematics}

Consider a final state splitting of a massless mother parton to massless daughter partons 1,2 and 3 as illustrated in figure 5. We use $(+,-, \perp)$ coordinates defined as in section 2.4, based on reference directions $u_{\mathrm{J}}$ and $n_{\mathrm{J}}$ with $u_{\mathrm{J}}+n_{\mathrm{J}}$ proportional to the fixed vector $Q_{0}$. We think of the partons as part of a jet with momentum components

$$
p_{\mathrm{J}} \approx P u_{\mathrm{J}}=(P, 0, \mathbf{0}) .
$$

For any of the individual splittings in figure 5, if we label the daughter partons as $i$ and $j$, we take the $(+,-, \perp)$ components of the momenta of partons to be

$$
\begin{aligned}
& p_{i}=\left(x_{i} P, \frac{\boldsymbol{p}_{i}^{2}}{2 x_{i} P}, \boldsymbol{p}_{i}\right), \\
& p_{j}=\left(x_{j} P, \frac{\boldsymbol{p}_{j}^{2}}{2 x_{j} P}, \boldsymbol{p}_{j}\right),
\end{aligned}
$$

as in eq. (3.2). Partons $i$ and $j$ may have daughter partons and thus have nonzero virtualities, but we are neglecting their virtualities here. We can define a squared transverse momentum $k_{\mathrm{T}}^{2}$ for the splitting by

$$
k_{\mathrm{T}}^{2}=\frac{1}{\left(x_{i}+x_{j}\right)^{2}}\left(x_{j} \boldsymbol{p}_{i}-x_{i} \boldsymbol{p}_{j}\right)^{2} .
$$

This matches the definition in eq. (2.8). 
One can use this $k_{\mathrm{T}}^{2}$ as the ordering variable for a shower. Note, however, that this is not close to the $k_{\mathrm{T}}^{2}$ definitions used in Pythia or Sherpa. It is, instead, close to the $k_{\mathrm{T}}^{2}$ variable used in the $\boldsymbol{k}_{\mathrm{T}}$ jet algorithm. See the discussion in section 2.4. Our purpose in this section is to see where ordering using this $k_{\mathrm{T}}^{2}$ gives a different set of shower histories than the ordering using $\Lambda^{2}$. The virtuality of the mother parton is related to $k_{\mathrm{T}}^{2}$ by

$$
\left(p_{i}+p_{j}\right)^{2}=\frac{\left(x_{i}+x_{j}\right)^{2}}{x_{i} x_{j}} k_{\mathrm{T}}^{2} .
$$

Ordering according to shower time as defined in eq. (3.13) amounts to ordering in the variable

$$
\Lambda^{2}=\frac{\left(p_{i}+p_{j}\right)^{2}}{x_{i}+x_{j}} \frac{Q_{0}^{2}}{2 p_{\mathrm{J}} \cdot Q_{0}} .
$$

The two ordering variables are related by

$$
\Lambda^{2}=\frac{x_{i}+x_{j}}{x_{i} x_{j}} k_{\mathrm{T}}^{2} \frac{Q_{0}^{2}}{2 p_{\mathrm{J}} \cdot Q_{0}} .
$$

Consider now the shower history $A$ in figure 5. We take $P$ to represent the plusmomentum of the mother parton, so that $x_{1}+x_{2}+x_{3}=1$. There are two splittings, which we can call I and II. The $k_{\mathrm{T}}$ values for the two splittings are

$$
\begin{aligned}
k_{\mathrm{T}}^{2}(\mathrm{I}) & =\left(\left(x_{1}+x_{2}\right) \boldsymbol{p}_{3}-x_{3}\left(\boldsymbol{p}_{1}+\boldsymbol{p}_{2}\right)\right)^{2}, \\
k_{\mathrm{T}}^{2}(\mathrm{II}) & =\frac{1}{\left(x_{1}+x_{2}\right)^{2}}\left(x_{1} \boldsymbol{p}_{2}-x_{2} \boldsymbol{p}_{1}\right)^{2} .
\end{aligned}
$$

It will prove useful to exchange the transverse momentum variables for two dimensional angular variables, defined by

$$
\boldsymbol{\theta}_{i}=\frac{\sqrt{2}}{P} \frac{\boldsymbol{p}_{i}}{x_{i}}
$$

We define differences in angles by

$$
\boldsymbol{\theta}_{i j}=\boldsymbol{\theta}_{i}-\boldsymbol{\theta}_{j} .
$$

Using these angular variables, the $k_{\mathrm{T}}^{2}$ values for the two splittings are

$$
\begin{aligned}
k_{\mathrm{T}}^{2}(\mathrm{I}) & =\frac{\left(p_{J} \cdot Q_{0}\right)^{2}}{Q_{0}^{2}} x_{3}^{2}\left(x_{1} \boldsymbol{\theta}_{31}+x_{2} \boldsymbol{\theta}_{32}\right)^{2}, \\
k_{\mathrm{T}}^{2}(\mathrm{II}) & =\frac{\left(p_{J} \cdot Q_{0}\right)^{2}}{Q_{0}^{2}} \frac{\left(x_{1} x_{2}\right)^{2}}{\left(x_{1}+x_{2}\right)^{2}} \boldsymbol{\theta}_{12}^{2} .
\end{aligned}
$$

The corresponding $\Lambda^{2}$ values are

$$
\begin{aligned}
\Lambda^{2}(\mathrm{I}) & =\frac{p_{J} \cdot Q_{0}}{2} \frac{x_{3}}{x_{1}+x_{2}}\left(x_{1} \boldsymbol{\theta}_{31}+x_{2} \boldsymbol{\theta}_{32}\right)^{2}, \\
\Lambda^{2}(\mathrm{II}) & =\frac{p_{J} \cdot Q_{0}}{2} \frac{x_{1} x_{2}}{x_{1}+x_{2}} \boldsymbol{\theta}_{12}^{2} .
\end{aligned}
$$

Thus history $\mathrm{A}$ is a valid shower history for partons 1,2 , and 3 according to $k_{\mathrm{T}}$ ordering if $k_{\mathrm{T}}^{2}(\mathrm{I})>k_{\mathrm{T}}^{2}$ (II) and it is a valid history according to $\Lambda$ ordering if $\Lambda^{2}(\mathrm{I})>\Lambda^{2}$ (II). The same analysis applies to histories B and C. We simply have to permute the labels 1,2,3 in eqs. (4.10) and (4.11). 


\subsection{A specific case}

Mostly, $\Lambda$ ordering and $k_{\mathrm{T}}$ ordering are equivalent for splittings to make three partons. Here is one case where they differ. Suppose that $x_{3} \ll x_{2} \ll 1$. Then we take $x_{1} \approx 1$ since $x_{1}+x_{2}+x_{3}=1$. Define $\boldsymbol{\theta}=\left(\boldsymbol{\theta}_{31}+\boldsymbol{\theta}_{32}\right) / 2$ and suppose that $\boldsymbol{\theta}_{12}^{2} \ll \boldsymbol{\theta}^{2}$. Then $\boldsymbol{\theta}_{31}^{2} \approx \boldsymbol{\theta}_{32}^{2} \approx \boldsymbol{\theta}^{2}$. Under these circumstances, for history A we have

$$
\begin{aligned}
k_{\mathrm{T}}^{2}(\mathrm{I}) & =\frac{\left(p_{J} \cdot Q_{0}\right)^{2}}{Q_{0}^{2}} x_{3}^{2} \boldsymbol{\theta}^{2}, \\
k_{\mathrm{T}}^{2}(\mathrm{II}) & =\frac{\left(p_{J} \cdot Q_{0}\right)^{2}}{Q_{0}^{2}} x_{2}^{2} \boldsymbol{\theta}_{12}^{2}
\end{aligned}
$$

and

$$
\begin{aligned}
\Lambda^{2}(\mathrm{I}) & =\frac{p_{J} \cdot Q_{0}}{2} x_{3} \boldsymbol{\theta}^{2}, \\
\Lambda^{2}(\mathrm{II}) & =\frac{p_{J} \cdot Q_{0}}{2} x_{2} \boldsymbol{\theta}_{12}^{2} .
\end{aligned}
$$

Thus history $\mathrm{A}$ is allowed for $k_{\mathrm{T}}$ ordering if

$$
\frac{\boldsymbol{\theta}_{12}^{2}}{\boldsymbol{\theta}^{2}}<\frac{x_{3}^{2}}{x_{2}^{2}}
$$

while history $\mathrm{A}$ is allowed for $\Lambda$ ordering if

$$
\frac{\boldsymbol{\theta}_{12}^{2}}{\boldsymbol{\theta}^{2}}<\frac{x_{3}}{x_{2}}
$$

Suppose that

$$
\frac{x_{3}^{2}}{x_{2}^{2}} \ll \frac{\boldsymbol{\theta}_{12}^{2}}{\boldsymbol{\theta}^{2}} \ll \frac{x_{3}}{x_{2}} .
$$

Then history $\mathrm{A}$ is allowed for $\Lambda$ ordering. However, history $\mathrm{A}$ is not generated with $k_{\mathrm{T}}$ ordering. With the same sort of analysis, we find instead that with $k_{\mathrm{T}}$ ordering we generate histories $\mathrm{B}$ and $\mathrm{C}$, while with $\Lambda$ ordering histories $\mathrm{B}$ and $\mathrm{C}$ are forbidden.

How can we interpret this result? The region in which $x_{3} \ll x_{2} \ll 1$ and $\boldsymbol{\theta}_{12}^{2} \ll \boldsymbol{\theta}^{2} \ll 1$ with $\boldsymbol{\theta}_{12}^{2} / \boldsymbol{\theta}^{2}$ limited by eq. (4.16) is important. It can generate four large logarithms, two from two angle integrations and two from two momentum fraction integrations. The analysis of section 3.1 indicates that both denominators in the Feynman graph corresponding to history A become independently small in this region, so that the square of graph A (in a physical gauge) gives a leading contribution. However, this does not work for graphs B and C. For instance, in graph $\mathrm{B}$, the $\left(p_{1}+p_{3}\right)^{2}$ is so large that it dominates the denominator proportional to $\left(p_{1}+p_{2}+p_{3}\right)^{2}$ as represented in eq. (3.4). Then the needed sensitivity to $p_{2}$ is absent. Thus the leading Feynman graph in the amplitude for $0 \rightarrow 1+2+3$ is that corresponding to history A.

What happens, then, if one uses a $k_{\mathrm{T}}$ ordered shower, which generates the shower using histories $\mathrm{B}$ and $\mathrm{C}$ but not $\mathrm{A}$ ? Because of color coherence, we get the right answer. There are two reasons for this. 
The first reason concerns the propagator denominator corresponding to splitting I in graphs $\mathrm{B}$ and $\mathrm{C}$. Consider graph $\mathrm{B}$. The momentum $p_{1}+p_{3}$ is really significantly off shell, considering that the corresponding parton subsequently splits to partons 1 and 3 . However, when we generate it, we treat it as exactly on shell. Thus when we generate splitting I in history B we use essentially the same propagator denominator as when we generate splitting II in history A. (However, depending on how the shower algorithm treats momentum conservation, the angle between partons 1 and 2 may be adjusted significantly to account for the recoil from parton 3.)

The second reason concerns the amplitude for splitting II in graph B, in which parton 3 is emitted. This is an emission of a soft, wide angle gluon. The amplitude can be approximated using the eikonal approximation,

$$
\mathcal{A}=\frac{g u_{\mathrm{J}} \cdot \epsilon_{3}}{u_{\mathrm{J}} \cdot p_{3}}
$$

where $u_{\mathrm{J}}$ is the lightlike vector in the direction of the jet, $u_{\mathrm{J}}=(1,0,0,0)$. Here $\epsilon_{3}$ is the polarization vector of gluon 3 . The same approximation applies to splitting II in graph C. Finally, this same approximation applies to splitting I in graph A. Thus the kinematic factor describing the emission of parton 3 is the same in graphs A, B, and C. The three graphs have different color factors, which we may call $\mathcal{C}_{A}, \mathcal{C}_{B}$, and $\mathcal{C}_{C}$, respectively. Color invariance implies that

$$
\mathcal{C}_{B}+\mathcal{C}_{C}=\mathcal{C}_{A}
$$

Putting these two arguments together, we see that when we add the parton shower approximations corresponding to histories $\mathrm{B}$ and $\mathrm{C}$, we get the parton shower approximation corresponding to history $\mathrm{A}$. That is, even though using a $k_{\mathrm{T}}$ ordered shower generates the given three parton configuration according to histories $\mathrm{B}$ and $\mathrm{C}$ instead of $\mathrm{A}$, the result is approximately the same.

An analogous argument shows that one can also use angular ordering, in which history A applies whenever $\boldsymbol{\theta}_{12} \ll \boldsymbol{\theta}$. This, of course, is the basic physics argument behind angle ordered showers $[19,20]$, as discussed in section 2.1.

To summarize, this example shows that the choice of shower ordering variable makes a difference in what shower histories are generated, but that because of color coherence for wide angle soft gluon emissions, this choice is less important than might be expected. However, this does not demonstrate that the choice of shower ordering variable makes no difference to the calculation of physical observables.

\section{Consequences for initial state splittings}

We now turn to the effect of using $\Lambda$ ordering in the initial state shower. We consider the case in which all of the partons are gluons. We seek insight into what initial state emissions are allowed by $\Lambda$ ordering. 


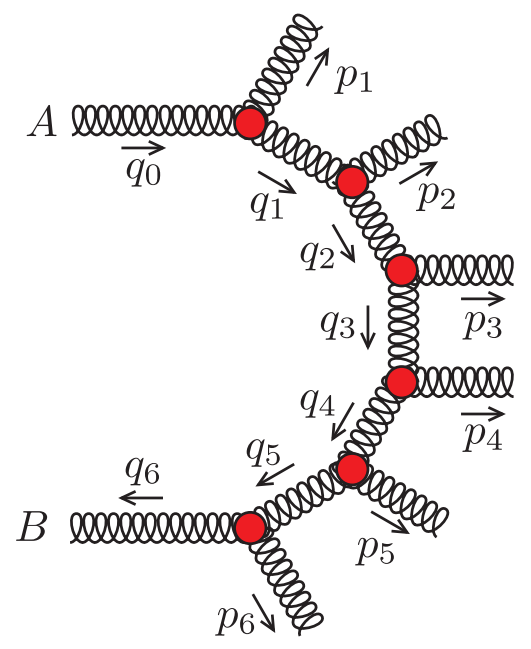

Figure 6. Initial state shower history showing initial state gluons with momenta $\left\{q_{0}, \ldots, q_{N}\right\}$ and emitted gluons with momenta $\left\{p_{1}, \ldots, p_{N}\right\}$ with $N=6$.

\section{$5.1 \quad$ Kinematics}

The kinematics for the initial state shower is illustrated in figure 6 . We have a sequence of initial state parton momenta, which we denote by

$$
\left\{q_{0}, q_{1}, \ldots, q_{h-1}, q_{h}, q_{h+1}, \ldots, q_{N-1}, q_{N}\right\} .
$$

Gluon 0 is the constituent of hadron A at the very end of the shower as we move from hard to soft interactions and gluon $N$ is the constituent of hadron B at the soft end of the initial state shower. The hard interaction is associated with the exchange of a gluon with momentum $q_{h}$.

As in section 3.3, we treat the hadron momenta as being lightlike: $p_{\mathrm{A}}^{2}=p_{\mathrm{B}}^{2}=0$. Then $s=\left(p_{\mathrm{A}}+p_{\mathrm{B}}\right)^{2}$ is given by $s=2 p_{\mathrm{A}} \cdot p_{\mathrm{B}}$. We use a reference frame in which $p_{\mathrm{A}}$ has only a plus component, $p_{\mathrm{A}}^{+}>0$, with $p_{\mathrm{A}}^{-}=0$ and $p_{\mathrm{A}}^{\perp}=0$. Similarly, $p_{\mathrm{B}}$ has only a minus component, $p_{\mathrm{B}}^{-}>0$, with $p_{\mathrm{B}}^{+}=0$ and $p_{\mathrm{B}}^{\perp}=0$.

We treat all of the momenta $p_{i}$ of emitted gluons as being on shell: $p_{i}^{2}=0$. We take initial state gluon 0 to be on shell and collinear with hadron A: $q_{0}=\eta_{0} p_{\mathrm{A}}$. We take initial state gluon $N$ to be on shell and collinear with hadron B: $-q_{N}=\xi_{N} p_{\mathrm{B}}$. Momentum is conserved at each vertex: $q_{i-1}=q_{i}+p_{i}$. Then the $q_{i}$ for $0<i<N$ are spacelike and generally have non-zero transverse components.

This is not the way that the shower is generated. In actually generating the initial state shower, at each splitting stage, we treat the new initial state gluon as being on shell with zero transverse momentum. This involves some approximations, which we outline in section 5.3. In this section, we ignore these complications and simply use spacelike initial state partons with non-zero transverse components and exact momentum conservation.

For each initial state gluon, decompose $q_{i}$ according to

$$
q_{i}=\eta_{i} p_{A}-\xi_{i} p_{B}+q_{i}^{\perp}
$$


where $q_{i}^{\perp} \cdot p_{\mathrm{A}}=q_{i}^{\perp} \cdot p_{\mathrm{B}}=0$. Because $p_{i} \cdot p_{\mathrm{A}}>0$ and $p_{i} \cdot p_{\mathrm{B}}>0$, we have $\eta_{i}>\eta_{i+1}$ and $\xi_{i}>\xi_{i-1}$. Also $\xi_{0}=0$ and $\eta_{N}=0$. That is, the momentum fractions $\eta_{i}$ decrease to zero as we move from hadron $\mathrm{A}$ to hadron $\mathrm{B}$ and the momentum fractions $\xi_{i}$ decrease to zero as we move from hadron B to hadron A. The initial state gluon momenta for $0<i<N$ are all spacelike:

$$
q_{i}^{2}=-\eta_{i} \xi_{i} s-\boldsymbol{q}_{i}^{2}<0 .
$$

Here and in the following, we denote the two components of $q_{i}^{\perp}$ with a boldface symbol $\boldsymbol{q}_{i}$, using the euclidian inner product in the transverse space so that $\boldsymbol{q}_{i}^{2}>0$.

\subsection{Evolution of the gluon virtualities}

There is an instructive relation between $q_{i}^{2}$ and $q_{i-1}^{2}$. To derive this relation, we start with the identity

$$
0<\left[\left(\eta_{i-1}-\eta_{i}\right) \boldsymbol{q}_{i}-\eta_{i} \boldsymbol{p}_{i}\right]^{2} .
$$

Using $\boldsymbol{q}_{i-1}=\boldsymbol{q}_{i}+\boldsymbol{p}_{i}$, this identity is equivalent to

$$
\frac{\boldsymbol{q}_{i-1}^{2}}{\eta_{i-1}}<\frac{\boldsymbol{q}_{i}^{2}}{\eta_{i}}+\frac{\boldsymbol{p}_{i}^{2}}{\eta_{i-1}-\eta_{i}} .
$$

The condition that $p_{i}$ is lightlike is

$$
\boldsymbol{p}_{i}^{2}=\left(\eta_{i-1}-\eta_{i}\right)\left(\xi_{i}-\xi_{i-1}\right) s .
$$

Thus eq. (5.4) is equivalent to

$$
\frac{\boldsymbol{q}_{i-1}^{2}+\eta_{i-1} \xi_{i-1} s}{\eta_{i-1}}<\frac{\boldsymbol{q}_{i}^{2}+\eta_{i} \xi_{i} s}{\eta_{i}} .
$$

Using eq. (5.2), this is

$$
\frac{-q_{i-1}^{2}}{\eta_{i-1}}<\frac{-q_{i}^{2}}{\eta_{i}} .
$$

This is the $\Lambda$ ordering condition (3.25) for an initial state shower starting at the hard interaction and moving toward hadron A except that "«" is replaced by " $<$." However, the actual shower generation uses " $<$."

With an analogous derivation, we have

$$
\frac{-q_{i+1}^{2}}{\xi_{i+1}}<\frac{-q_{i}^{2}}{\xi_{i}} .
$$

This is the $\Lambda$ ordering condition for an initial state shower starting at the hard interaction and moving toward hadron B.

We thus see that shower splittings that violate $\Lambda$ ordering in the form (3.25) are impossible if we use exact kinematics in the initial state shower. However, we do not use exact kinematics. Instead, we approximate initial state partons as being exactly on shell, then adjust them to have spacelike momenta by taking momentum from elsewhere in the event, as described in the following subsection. When we make these approximations, it is important to impose $\Lambda$ ordering so that we do not generate splittings that invalidate the approximations. 


\subsection{Momentum conservation}

The initial state shower does not appear all at once as depicted in figure 6 . Rather, it is generated step by step, starting from the hard interaction. It will be important for us to understand the relationship between the final result depicted in figure 6 and the steps in generating the shower. To this end, us suppose that the shower has been partly generated. At the current stage of shower generation, the latest parton to be generated on the hadron A side is parton $i$, with $i<h$. The latest parton to be generated on the hadron B side is parton $j$, with $j>h$. We work in a reference frame, "frame 0 ," in which these partons have zero transverse momenta. We approximate them as being on shell. That is, we are neglecting their virtualities as having negligible effect on the calculation of all harder interactions. Thus partons $i$ and $j$ have momentum components

$$
\begin{aligned}
q_{i} & =\left(\eta_{i} p_{\mathrm{A}}^{+}, 0, \mathbf{0}\right) & & \text { frame } 0, \\
-q_{j} & =\left(0, \xi_{j} p_{\mathrm{B}}^{-}, \mathbf{0}\right) & & \text { frame } 0 .
\end{aligned}
$$

Now we let parton $i$ split. Unfortunately, a parton with zero virtuality cannot split. Thus we need to change $q_{i}$ to a new momentum $\tilde{q}_{i}$ with

$$
\tilde{q}_{i}=\left(\tilde{\eta}_{i} p_{\mathrm{A}}^{+}, \frac{\tilde{q}_{i}^{2}}{2 \tilde{\eta}_{i} p_{\mathrm{A}}^{+}}, \mathbf{0}\right) \quad \text { frame } 0 .
$$

We want the shower to exactly conserve momentum, so we will have to take the needed momentum $\tilde{q}_{i}-q_{i}$ from elsewhere in the event. In DEDUCTOR, get the needed momentum by applying a small Lorentz transformation $\Lambda_{1}(\omega)$ to every final state parton that exists at this stage of the evolution,

$$
p_{k} \rightarrow \tilde{p}_{k}=\Lambda_{1}(\omega) p_{k} \quad k=i+1, \ldots, j .
$$

Then if we define

$$
Q_{i j}=\sum_{k=i+1}^{j} p_{k}
$$

we have

$$
Q_{i j} \rightarrow \widetilde{Q}_{i j}=\Lambda_{1}(\omega) Q_{i j}
$$

The original $Q_{i j}$ obeys

$$
q_{i}=Q_{i j}+q_{j} .
$$

If we now use momentum conservation with the shifted final state momenta, we have a modified $q_{i}$,

$$
\tilde{q}_{i}=\tilde{Q}_{i j}+q_{j} .
$$

That is

$$
\begin{aligned}
\tilde{q}_{i} & =\Lambda_{1}(\omega) Q_{i j}+q_{j} \\
& =\Lambda_{1}(\omega)\left[q_{i}-q_{j}\right]+q_{j} \\
& =\Lambda_{1}(\omega) q_{i}+\left(1-\Lambda_{1}(\omega)\right) q_{j} .
\end{aligned}
$$


We choose the Lorentz transformation $\Lambda_{1}(\omega)$ to be a boost in the $z$-direction with boost angle $\omega$. Then the components of $\tilde{q}_{i}$ are

$$
\tilde{q}_{i}=\left(e^{\omega} \eta_{i} p_{\mathrm{A}}^{+},-\left(1-e^{-\omega}\right) \xi_{j} p_{\mathrm{B}}^{-}, \mathbf{0}\right) .
$$

With this, we obtain the square of $\tilde{q}_{i}$ as a function of $\omega$,

$$
\begin{aligned}
-\tilde{q}_{i}^{2} & =\left(e^{\omega}-1\right) 2 \eta_{i} \xi_{j} p_{\mathrm{A}} \cdot p_{\mathrm{B}} \\
& =\left(e^{\omega}-1\right) Q_{i j}^{2},
\end{aligned}
$$

where we have used eq. (5.15). Thus

$$
e^{\omega}=1+\frac{-\tilde{q}_{i}^{2}}{Q_{i j}^{2}}
$$

Since the virtuality of any exchanged gluon is typically much smaller than the square of the momenta of all of the final state particles created between $i$ and $j$, we need only a small boost: $\omega \ll 1$. We see that the momentum fraction $\tilde{\eta}_{i}$ in eq. (5.10) is slightly different from $\eta_{i}$ :

$$
\tilde{\eta}_{i}=e^{\omega} \eta_{i}
$$

Now that gluon $i$ has a nonzero virtuality, it can split in backward evolution to a new initial state gluon with momentum $q_{i-1}$ and a final state gluon with momentum $p_{i}$, with

$$
q_{i-1}-p_{i}=\tilde{q}_{i}
$$

where $\tilde{q}_{i}$ is given by eq. (5.10). We take $q_{i-1}$ and $p_{i}$ to be lightlike with components

$$
\begin{aligned}
q_{i-1} & =\left(\eta_{i-1} p_{\mathrm{A}}^{+}, \frac{\boldsymbol{k}_{i}^{2}}{2 \tilde{z}_{i}^{2} \eta_{i-1} p_{\mathrm{A}}^{+}}, \frac{1}{\tilde{z}_{i}} \boldsymbol{k}_{i}\right) & & \text { frame } 0 \\
p_{i} & =\left(\left(1-\tilde{z}_{i}\right) \eta_{i-1} p_{\mathrm{A}}^{+}, \frac{\boldsymbol{k}_{i}^{2}}{2 \tilde{z}_{i}^{2}\left(1-\tilde{z}_{i}\right) \eta_{i-1} p_{\mathrm{A}}^{+}}, \frac{1}{\tilde{z}_{i}} \boldsymbol{k}_{i}\right) & & \text { frame } 0 .
\end{aligned}
$$

Here $q_{i-1}$ has a new momentum fraction $\eta_{i-1}$. We define

$$
\tilde{z}_{i}=\frac{\tilde{\eta}_{i}}{\eta_{i-1}}
$$

and assign a momentum fraction $\left(1-\tilde{z}_{i}\right) \eta_{i-1}$ to the emitted gluon. Then the plus component of momentum is conserved according to eq. (5.21). We assign transverse momentum $\boldsymbol{k}_{i} / \tilde{z}_{i}$ to both $q_{i-1}$ and $p_{i}$. Then the transverse components of momentum are also conserved. With a small calculation, we see that the minus component of momentum is conserved as long as $\boldsymbol{k}_{i}$ is chosen so that

$$
-\tilde{q}_{i}^{2}=\frac{\boldsymbol{k}_{i}^{2}}{1-\tilde{z}_{i}} .
$$

We need one more step. We should change to a new reference frame, "frame 1," so that the transverse components of $q_{i-1}$ are zero. This is simple with a null-plane boost 
$\Lambda_{2}(\boldsymbol{v})$ such that a vector with components $P$ in frame 0 has components $P^{\prime}=\Lambda_{2}(\boldsymbol{v}) P$ in frame 1 , with

$$
P^{\prime}=\left(P^{+}, P^{-}+\frac{1}{2} P^{+} \boldsymbol{v}^{2}+\boldsymbol{P} \cdot \boldsymbol{v}, \boldsymbol{P}+P^{+} \boldsymbol{v}\right)
$$

If we choose

$$
\boldsymbol{v}=-\frac{1}{\tilde{\eta}_{i} p_{\mathrm{A}}^{+}} \boldsymbol{k}_{i}
$$

we have

$$
\begin{aligned}
q_{i-1} & =\left(\eta_{i-1} p_{\mathrm{A}}^{+}, 0, \mathbf{0}\right) & & \text { frame } 1, \\
p_{i} & =\left(\left(1-\tilde{z}_{i}\right) \eta_{i-1} p_{\mathrm{A}}^{+}, \frac{\boldsymbol{k}_{i}^{2}}{2\left(1-\tilde{z}_{i}\right) \eta_{i-1} p_{\mathrm{A}}^{+}}, \boldsymbol{k}_{i}\right) & & \text { frame } 1, \\
\tilde{q}_{i} & =\left(\tilde{\eta}_{i} p_{\mathrm{A}}^{+}, \frac{\tilde{q}_{i}^{2}}{2 \eta_{i-1} p_{\mathrm{A}}^{+}},-\boldsymbol{k}_{i}\right) & & \text { frame } 1, \\
-q_{j} & =\left(0, \xi_{j} p_{B}^{-}, \mathbf{0}\right) & & \text { frame } 1 .
\end{aligned}
$$

The components of the final state momenta $\tilde{p}_{i}$ for $i=i+1, \ldots, j$ change according to the boost defined by eq. (5.25). However, the boost does not change the components of a vector that lies entirely in the minus direction, so the components of $q_{j}$ are not changed.

The practical effect of this is that $q_{i-1}$ and $p_{i}$ are given by eq. (5.27), while the components of the momenta $p_{k}$ of final state gluons are modified by the net Lorentz transformation $\Lambda_{2}(\boldsymbol{v}) \Lambda_{1}(\omega)$. This analysis has been for massless partons. We state the needed Lorentz transformation for the general case of massive partons in appendix A.

The important point of this subsection is not the precise form of the Lorentz transformations, but rather the idea that, in generating an initial state splitting, we first take a small amount of momentum from the final state that allows us to conserve momentum in the splitting, then we change reference frames so that the new initial state parton has zero transverse momentum. Thus the momenta of final state partons shift at each emission so as to recoil against the transverse momentum of a newly emitted parton.

\subsection{Dynamical regimes}

Now, consider possible dynamical regimes for the initial state shower of figure 6 . One regime is the standard one with soft or collinear gluons emitted from the initial state gluons. For the hadron $\mathrm{A}$ side of the shower, the momentum fractions $z_{i}=\eta_{i} / \eta_{i-1}$ are either close to 1 for a soft gluon emission or finite, close to neither 0 or 1 , for a collinear splitting. Then all of the $\eta_{i}$ are roughly the same size. Then eq. (5.7) implies that the virtualities $-q_{i}^{2}$ decrease as we move from the hard interaction toward hadron A. If the $z_{i}$ are not close to 1 , the splitting transverse momenta,

$$
\boldsymbol{k}_{i}=\left(1-z_{i}\right) \boldsymbol{q}_{i-1}-\boldsymbol{p}_{i}
$$

also decrease since, from eq. (3.23),

$$
\boldsymbol{k}_{i}^{2} \approx\left(1-z_{i}\right)\left(-q_{i}^{2}\right) .
$$


There is another regime, which one can call the cut pomeron regime. All of the $\boldsymbol{k}_{i}$ can be of roughly the same size, but the $z_{i}$ can be small: $\eta_{i-1} \gg \eta_{i}$. In this regime, also $\xi_{i+1} \gg \xi_{i}$. The cut pomeron regime can be important when $s=2 p_{\mathrm{A}} \cdot p_{\mathrm{B}}$ is much larger that the $\boldsymbol{k}_{i}^{2}$. This regime has been extensively studied [39-42] and there are computer codes that generate events in the cut pomeron regime [43-48]. Of course, there are possible regimes intermediate between the standard regime and the cut pomeron regime, but we content ourselves here with just these two idealized cases.

Since neither transverse momenta nor virtualities need to decrease during shower generation if splitting momentum fractions $z_{i}$ are small, DEDUCtor can generate events in the cut pomeron regime. Evidently, this is very different from what is allowed to happen in a $k_{\mathrm{T}}$ ordered shower (with $k_{\mathrm{T}}$ defined as above) or a virtuality ordered shower.

However, there is one issue that we need to consider in Deductor. We start with a hard interaction, but in figure 6 , which is the hard interaction? It seems that we can start anywhere. We adopt the following procedure. Of all the initial state propagators in figure 6 , one has the largest $\left|q_{i}^{2}\right|$. We take that to be the hard interaction, labelled $h$. Then we develop initial state showers on both the A side and the B side of the hard interaction. We want the gluon with $i=h$ to be the one with the largest virtuality, so we require

$$
\left|q_{i}^{2}\right|<\left|q_{h}^{2}\right|
$$

in the initial state shower splittings. In figure 6 as drawn, $q_{h}^{2}$ is the invariant $\hat{t}$ of the parton-parton scattering that constitutes the hard interaction. However, in generating the hard interaction, there is no distinction between the two final state partons. If we exchange them, $q_{h}^{2}$ is the invariant $\hat{u}$. Thus we define

$$
\left|q_{h}^{2}\right|=\min (|\hat{t}|,|\hat{u}|) .
$$

Note that applying the cut eq. (5.30) is by no means the same as imposing $k_{\mathrm{T}}$ ordering or virtuality ordering on the initial state shower. No $\left|q_{i}^{2}\right|$ can be larger than $\left|q_{h}^{2}\right|$, but as we move from the hard interaction towards hadron $\mathrm{A}$ or towards hadron $\mathrm{B}$, the $\left|q_{i}^{2}\right|$ can decrease, then increase again. Thus we can have a gluon emitted with high transverse momentum and large negative rapidity, another gluon emitted with high transverse momentum and large positive rapidity, and several gluons emitted with smaller transverse momenta and intermediate rapidities.

Although Deductor does generate events in the cut pomeron regime, it was not designed with this regime in mind. To adapt it for cut pomeron physics, one would need to incorporate suitable Sudakov factors for each propagator in figure 7. These Sudakov factors should represent unresolved real radiation together with virtual radiation. In the current version of Deductor, there is no Sudakov factor associated with the hard interaction and the factors associated with initial state gluon emissions are merely those that keep the hard scattering cross section from being changed by shower evolution. It would be fairly straightforward to create extra Sudakov factors as weights that would differ from 1 in the event of large rapidity separations. Investigation of what extra Sudakov factors to incorporate remains as a topic for future investigation. 


\subsection{A numerical investigation}

In order to understand the effect in DeDUCTOR of initial state parton splittings with $z \ll 1$ and of very forward gluon scattering in the hard interaction, we examine an observable that was calculated in ref. [47] using the generator High EnERGY Jets. We examine multijet production in $7 \mathrm{TeV}$ proton-proton collisions. The jets are defined using the anti$k_{\mathrm{T}}$ algorithm [49] with $\mathrm{R}=0.6$, found with the aid of FASTJET [50]. Only jets with $\left|\boldsymbol{p}_{i \perp}\right|>30 \mathrm{GeV}$ and rapidity $\left|y_{j}\right|<4.5$ are considered. Define $\Delta y=y_{\mathrm{f}}-y_{\mathrm{b}}$, where $y_{\mathrm{f}}$ is the rapidity of the jet with the greatest rapidity and $y_{\mathrm{b}}$ is the rapidity of the jet with the smallest rapidity. We consider only events in which the average of the transverse momenta of the most forward and backward jets is sufficiently large: $\left(\left|\boldsymbol{p}_{\mathrm{f} \perp}\right|+\left|\boldsymbol{p}_{\mathrm{b} \perp}\right|\right) / 2>60 \mathrm{GeV}$. In these events, we measure the total number $N$ of jets. In standard events, the hard scattering is gluon-gluon scattering to produce jets with $\left|\boldsymbol{p}_{\perp}\right|>60 \mathrm{GeV}$. Then $N$ should usually be 2 . Initial state radiation can produce a $30 \mathrm{GeV}$ jet in the rapidity range between the jets produced by the hard scattering, but this should be rather rare. In the cut pomeron picture, when $\Delta y$ is large, there is the possibility of producing extra jets in the rapidity range between the jets with extremal rapidities. Thus if we plot the average value $\langle N\rangle$ of the number of jets versus $\Delta y$, we can expect to see a rising curve.

Plots of $\langle N\rangle$ versus $\Delta y$ are shown in figure 7 . There are five curves. The lowest was generated with Deductor. With Deductor, the production of extra jets between the jets with extremal rapidities is not rare: the average number, $\langle N\rangle-2$, of extra jets rises with $\Delta y$ to a value of more than $1 / 2$. The next higher curve in this range was generated with Pythia (version 8.176) [11]. The Pythia curve is somewhat higher than the Deductor curve, although, since the PYтнIA shower is $k_{\mathrm{T}}$ ordered, one would think that the PүтніA curve should be lower. One effect that tends to raise the PүтніA curve is that the value of $\alpha_{\mathrm{S}}$ for initial state radiation is large: $\alpha_{\mathrm{s}}\left(M_{\mathrm{Z}}^{2}\right)=0.137$ compared to $\alpha_{\mathrm{S}}\left(M_{\mathrm{Z}}^{2}\right)=0.118$ in Deductor. ${ }^{8}$ The next three curves were generated by High Energy Jets and were taken from figure 11 of ref. [47]. The three curves correspond to three different choices of parameters within High Energy Jets. We see that the High Energy Jets curves lie substantially above the Pythia and Deductor curves.

By construction, Deductor allows the transverse momentum of produced jets to decrease and then increase as the initial state shower proceeds over a large rapidity range. The probability that this happens is controlled by Sudakov factors that are constructed to preserve the cross section for the hard event. To represent cut pomeron physics, this may not be what one wants. We see in figure 7 that although the $\Lambda$ ordering in Deductor allows the production of extra high $k_{\mathrm{T}}$ jets, the probability for doing so is not as high as it is in High EnERGy Jets. We expect that with implementation of more physically motivated Sudakov factors, the DEDUCTOR curve may be modified for large $\Delta y$.

\footnotetext{
${ }^{8}$ In Deductor, $\alpha_{\mathrm{s}}$ is evaluated at a scale $\lambda_{\mathrm{R}} k_{\mathrm{T}}^{2}$, where $\lambda_{\mathrm{R}} \approx 0.4$ [1]. Thus a more accurate comparison is to $\alpha_{\mathrm{s}}\left(\lambda_{\mathrm{R}} M_{\mathrm{Z}}^{2}\right)=0.126$ for Deductor.
} 


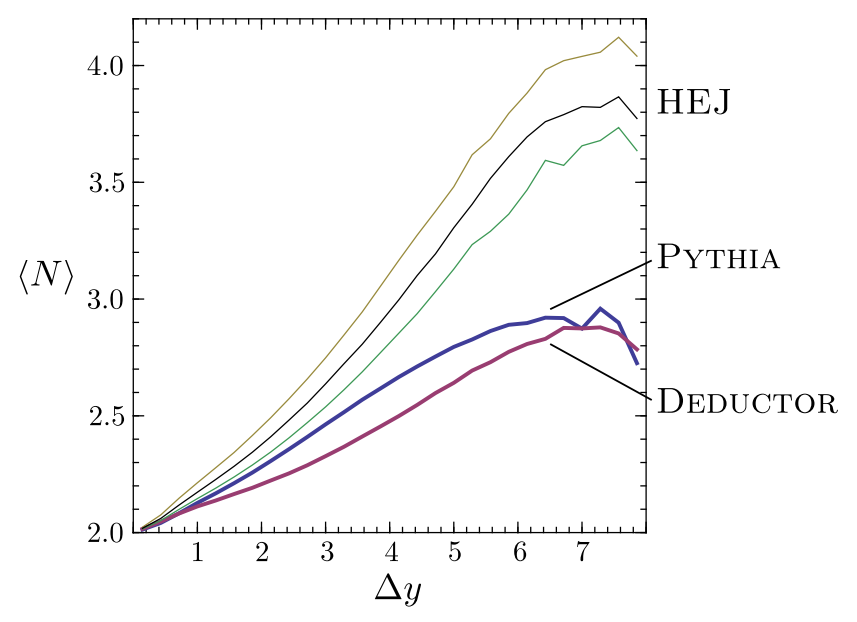

Figure 7. Average number $\langle N\rangle$ of jets versus the rapidity difference $\Delta y$ between the jet with the greatest rapidity and the jet with the least rapidity. All jets must have $\left|\boldsymbol{p}_{i \perp}\right|>30 \mathrm{GeV}$ and the average of the transverse momentum of the two jets with extremal rapidity must be at least $60 \mathrm{GeV}$. The upper three curves are $\langle N\rangle$ according to HIGH EnERGY JETS, with three choices within this program, as reported in figure 11 of ref. [47]. The middle curve in the range $5<\Delta y<7$ is the result from Pythia. The bottom curve in this range is the result from Deductor.

\section{Ending the shower}

The shower time that we use, eqs. (3.12) and (3.13), is well suited for determining the relative ordering of two splitting vertices. However, at some point in shower evolution, the splittings are too soft for perturbation theory to be reliable. Thus one needs to end the perturbative shower and substitute a non-perturbative model. ${ }^{9}$ The simplest thing to do would be to stop the shower at a fixed value of $\Lambda$. However, that is not sensible because of the factor $1 / p_{i} \cdot Q_{0}$ in $\Lambda^{2}$. This factor is not invariant under boosts in the direction of $p_{i}$. Consider potential splittings of two partons with the same virtuality $\left|p_{i}^{2}-m_{i}^{2}\right|$ and the same splitting variable $z$. With a fixed cutoff on $\Lambda$, a parton that is part of a moderate momentum jet might be allowed to split while a parton that is part of a very high momentum jet is not allowed to split.

Instead, we veto all splittings in which $k_{\mathrm{T}}^{2}<k_{T, \min }^{2}$ where $k_{T \text {,min }}^{2}$ is, say, $1 \mathrm{GeV} .{ }^{10}$ Neglecting all masses, $k_{\mathrm{T}}^{2}=z(1-z)\left|p_{i}^{2}\right|$ for final state splittings, so this cut keeps the virtuality, $z$, and $(1-z)$ from being too small. For initial state splittings with zero masses, $k_{\mathrm{T}}^{2}=(1-z)\left|q_{i}^{2}\right|$, so this cut keeps the virtuality and $(1-z)$ from being too small. Since the end of PyтhIA showers is based on $k_{\mathrm{T}}^{2}$, this choice facilitates matching to the PyтHIA model of hadronization.

\footnotetext{
${ }^{9}$ The shower in ref. [1] does not have a non-perturbative hadronization model. We anticipate providing an interface to the string model using PYTHIA.

${ }^{10}$ Here, to be precise, for a final state splitting, $\boldsymbol{k}_{\mathrm{T}}$ is the part of the momentum of one daughter parton relative to the mother parton. For an initial state splitting, $\boldsymbol{k}_{\mathrm{T}}$ is part of the momentum of the parton 2 in section 3.3 relative to the direction of initial state parton 1 . In each case, we use a reference frame in which $Q_{0}$ has only a time component.
} 


\section{Conclusions}

In a parton shower event generator, one assigns a variable $V_{i}^{2}$ to parton splittings for the purpose of ordering the splittings. If the splitting of parton $i$ comes before the splitting of parton $j$ then $V_{i}^{2}>V_{j}^{2}$. Often, as in Deductor, $V_{i}^{2}$ is related to the hardness of the splitting in the sense that $V_{i}^{2} \rightarrow 0$ when the daughter partons become collinear or when one becomes soft.

This hardness ordering criterion allows a rather wide range of choices for $V_{i}^{2}$. We propose a more restrictive criterion based on factoring soft interactions from hard interactions. Recall that when one wants to calculate an infrared safe inclusive cross section, one can factor the hardest interaction from parton distribution functions and from softer interactions. In our view, a parton shower event generator works the same way, but on many scales. First the hardest interaction is factored from the rest, then the next hardest interaction, and so forth. We ask that this work on a graph-by-graph basis in a physical gauge (to leading order in the splitting functions). With that demand, we find that $V_{i}^{2}$ should be $\Lambda_{i}^{2}$, where

$$
\begin{aligned}
\Lambda_{i}^{2} & =\frac{p_{i}^{2}-m_{i}^{2}}{2 p_{i} \cdot Q_{0}} Q_{0}^{2} & \text { final state parton } \\
\Lambda_{i}^{2} & =\frac{\left|p_{i}^{2}-m_{i}^{2}\right|}{2 \eta_{i} p_{\mathrm{A}} \cdot Q_{0}} Q_{0}^{2} & \text { initial state parton } .
\end{aligned}
$$

Here $\eta_{i}$ is the momentum fraction for an initial state parton from hadron $\mathrm{A}$ and $Q_{0}$ is a fixed timelike vector, which we take to be the sum of the momenta of the final state particles at the hardest interaction. We use this ordering parameter in Deductor.

We investigated in section 4 the relation of $\Lambda$ ordering to a version of $k_{\mathrm{T}}$ ordering, at least for the case of massless final state partons and for just two successive splittings. The ordering of vertices according to $\Lambda$ and the $k_{\mathrm{T}}$ that we use can be different in the case of wide angle emission of soft gluons. Of course, wide angle, soft gluon emission is important in a gauge theory like QCD. However, wide angle, soft gluon emission has important color coherence properties. Taking color coherence into account, we find that $k_{\mathrm{T}}$ ordered emissions, treated with the parton shower approximation of on-shell daughter partons and summed over graphs, gives approximately the same result as $\Lambda$ ordered emissions. Indeed, angle ordered parton showers, by design, also reorder emissions to get an equivalent result to hardness ordered emissions. Thus parton shower algorithms are surprisingly robust against changes of the ordering prescription, at least for final state splittings to the level that we have investigated the question. Nevertheless, we prefer the ordering parameter of eq. (7.1) because, with this choice, factorization works graph by graph.

We investigated in section 5 the relation of $\Lambda$ ordering to virtuality ordering or a version of $k_{\mathrm{T}}$ ordering for the initial state shower in hadron-hadron collisions. We found that $\Lambda$ ordering makes available a wider phase space in the case of a series of splittings with small values of the momentum fraction variable $z$. The wider phase space incorporates the phase space associated with cut pomeron exchange. We have not investigated the Sudakov factors that should be associated with such emissions. Currently in DeduCtor we use only the standard, probability conserving, Sudakov factors. Then we find that the additional phase space is not often filled. 


\section{Acknowledgments}

This work was supported in part by the United States Department of Energy and by the Helmoltz Alliance "Physics at the Terascale." We thank Jennifer Smillie and Jeppe Andersen for sending us results from High EnERGy JeTs. We thank Hannes Jung for helpful discussions about small $x$ effects.

\section{A Momentum conservation for initial state splitting}

We saw in section 5.3 how momentum conservation is maintained in generating the initial state shower when all partons are massless. In this appendix, we record the needed Lorentz transformation when the partons are allowed to have non-zero masses.

Let the momenta of the incoming hadrons, treated as massless, be $p_{\mathrm{A}}$ and $p_{\mathrm{B}}$, defined so that $s=2 p_{\mathrm{A}} \cdot p_{\mathrm{B}}$. Let the momentum fractions of the incoming partons be $\eta_{\mathrm{a}}$ and $\eta_{\mathrm{b}}$. The incoming partons, with flavors $a$ and $b$, may have non-zero masses, so we define their momenta to be

$$
\begin{aligned}
& p_{\mathrm{a}}=\eta_{\mathrm{a}} p_{\mathrm{A}}+\frac{m(a)^{2}}{\eta_{\mathrm{a}} \eta_{\mathrm{b}} s} \eta_{\mathrm{b}} p_{\mathrm{B}}, \\
& p_{\mathrm{b}}=\eta_{\mathrm{a}} p_{\mathrm{B}}+\frac{m(b)^{2}}{\eta_{\mathrm{a}} \eta_{\mathrm{b}} s} \eta_{\mathrm{a}} p_{\mathrm{A}} .
\end{aligned}
$$

We can simplify our notation by defining massless vectors

$$
\begin{aligned}
& n_{\mathrm{a}}=\eta_{\mathrm{a}} p_{\mathrm{A}}, \\
& n_{\mathrm{b}}=\eta_{\mathrm{a}} p_{\mathrm{B}},
\end{aligned}
$$

and dimensionless mass squared variables

$$
\nu(f)=\frac{m(f)^{2}}{\eta_{\mathrm{a}} \eta_{\mathrm{b}} s} .
$$

Then

$$
\begin{aligned}
& p_{\mathrm{a}}=n_{\mathrm{a}}+\nu(a) n_{\mathrm{b}}, \\
& p_{\mathrm{b}}=n_{\mathrm{b}}+\nu(b) n_{\mathrm{a}} .
\end{aligned}
$$

We can use the vectors $n_{\mathrm{a}}$ and $n_{\mathrm{b}}$ as two basis vectors. The projection operator onto the space transverse to $n_{\mathrm{a}}$ and $n_{\mathrm{b}}$ is given by

$$
g_{\perp}^{\mu \nu}=g^{\mu \nu}-\frac{n_{\mathrm{a}}^{\mu} n_{\mathrm{b}}^{\nu}+n_{\mathrm{b}}^{\mu} n_{\mathrm{a}}^{\nu}}{n_{\mathrm{a}} \cdot n_{\mathrm{b}}} .
$$

Now suppose that parton "a" splits, in the sense of backward evolution. The new incoming parton has flavor $\hat{a}$, mass $m(\hat{a})$, and momentum fraction $\hat{\eta}_{\mathrm{a}}$. We define the momentum fraction of the splitting as $z=\eta_{\mathrm{a}} / \hat{\eta}_{\mathrm{a}} \cdot{ }^{11}$ The momentum of the new initial state

\footnotetext{
${ }^{11}$ In generating the shower, we define the splitting variable $z=\eta_{\mathrm{a}} / \hat{\eta}_{\mathrm{a}}$. Elsewhere in this paper, we use $z$ to denote a momentum fraction ratio in several contexts. The precise value of $z$ depends on which partons are approximated as being on shell and which are allowed to be off shell. We hope that this does not cause confusion.
} 
parton is then

$$
\hat{p}_{\mathrm{a}}=\frac{1}{z} n_{\mathrm{a}}+z \nu(\hat{a}) n_{\mathrm{b}} .
$$

A new final state particle, labelled $m+1$, is emitted. Its momentum has the form

$$
\hat{p}_{m+1}=x_{\mathrm{a}} n_{\mathrm{a}}+x_{\mathrm{b}} n_{\mathrm{b}}+k_{\perp},
$$

where the magnitude of the transverse momentum is fixed by

$$
\left|k_{\perp}^{2}\right|=2 x_{\mathrm{a}} x_{\mathrm{b}} n_{\mathrm{a}} \cdot n_{\mathrm{b}}-m\left(f_{m+1}\right)^{2} .
$$

The azimuthal angle, $\phi$, of $k_{\perp}$ is another of the splitting variables besides $z$. The third splitting variable is the dimensionless virtuality

$$
y=-\frac{\left(\hat{p}_{\mathrm{a}}-\hat{p}_{m+1}\right)^{2}-m^{2}(a)}{2 n_{\mathrm{a}} \cdot n_{\mathrm{b}}} .
$$

Typically, $y \ll 1$.

In order to conserve momentum, we need to adjust the momenta of all of the previously created final state particles,

$$
p_{i}^{\mu} \rightarrow \hat{p}_{i}^{\mu}=\Lambda_{\nu}^{\mu} p_{i}^{\nu} \quad i=1, \ldots, m .
$$

This works provided

$$
\begin{aligned}
& x_{\mathrm{a}}=\frac{1 / z-1-y(1+z \nu(b))-(1-z) \nu(a) \nu(b)-z \nu\left(f_{m+1}\right) \nu(b)}{1-z^{2} \nu(\hat{a}) \nu(b)}, \\
& x_{\mathrm{b}}=z \frac{y+\nu\left(f_{m+1}\right)-\nu(a)+(1+y) z \nu(\hat{a})+z \nu(\hat{a}) \nu(b)(\nu(a)-z \nu(\hat{a}))}{1-z^{2} \nu(\hat{a}) \nu(b)} .
\end{aligned}
$$

The needed Lorentz transformation starts with a small boost along the $z$-axis

$$
\Lambda_{\|}^{\mu \nu}(\omega)=g_{\perp}^{\mu \nu}+\frac{e^{\omega} n_{\mathrm{a}}^{\mu} n_{\mathrm{b}}^{\nu}+e^{-\omega} n_{\mathrm{b}}^{\mu} n_{\mathrm{a}}^{\nu}}{n_{\mathrm{a}} \cdot n_{\mathrm{b}}},
$$

with boost angle $\omega$ given by

$$
e^{\omega}=[1+\nu(b)]^{-1}\left[\frac{1}{z}+\nu(b)-x_{\mathrm{a}}\right] .
$$

Then we apply a transverse null plane boost $\Lambda_{\perp}\left(v_{\perp}\right)$,

$$
\Lambda_{\perp}^{\mu \nu}\left(v_{\perp}\right)=g^{\mu \nu}+\sqrt{\frac{2}{n_{\mathrm{a}} \cdot n_{\mathrm{b}}}}\left[v_{\perp}^{\mu} n_{\mathrm{b}}^{\nu}-n_{\mathrm{b}}^{\mu} v_{\perp}^{\nu}\right]-v_{\perp}^{2} \frac{n_{\mathrm{b}}^{\mu} n_{\mathrm{b}}^{\nu}}{n_{\mathrm{a}} \cdot n_{\mathrm{b}}},
$$

with boost velocity

$$
v_{\perp}=-\frac{e^{-\omega}}{[1+\nu(b)] \sqrt{2 n_{\mathrm{a}} \cdot n_{\mathrm{b}}}} k_{\perp} .
$$

Our normalization convention for $v_{\perp}$ here is different from that used in section 5.3. We also note that $\Lambda\left(v_{\perp}\right)$ leaves $n_{\mathrm{b}}$ unchanged, but it changes $p_{\mathrm{b}}$ by a small amount, proportional to $\nu(b)$. Thus the interpretation is a little different from that described in section 5.3 for massless partons. However, the difference in interpretation is of no real consequence. 
The complete Lorentz transformation is

$$
\begin{aligned}
\Lambda^{\mu \nu}\left(\omega, v_{\perp}\right)= & g_{\perp}^{\mu \nu}+\frac{e^{\omega} n_{\mathrm{a}}^{\mu} n_{\mathrm{b}}^{\nu}+e^{-\omega} n_{\mathrm{b}}^{\mu} n_{\mathrm{a}}^{\nu}}{n_{\mathrm{a}} \cdot n_{\mathrm{b}}} \\
& +\sqrt{\frac{2}{n_{\mathrm{a}} \cdot n_{\mathrm{b}}}}\left[e^{\omega} v_{\perp}^{\mu} n_{\mathrm{b}}^{\nu}-n_{\mathrm{b}}^{\mu} v_{\perp}^{\nu}\right]-e^{\omega} v_{\perp}^{2} \frac{n_{\mathrm{b}}^{\mu} n_{\mathrm{b}}^{\nu}}{n_{\mathrm{a}} \cdot n_{\mathrm{b}}} .
\end{aligned}
$$

The analysis of this appendix follows from applying the choice $y, z, \phi$ of splitting variables to the analysis of section 4.4 of ref. [3] except that we choose the Lorentz transformation $\Lambda_{\nu}^{\mu}$ differently. As noted at eq. (3.9) of ref. [37], the choice that we make here is better adapted to summing logs to evaluate the transverse momentum of Z-bosons produced in the Drell-Yan process.

Open Access. This article is distributed under the terms of the Creative Commons Attribution License (CC-BY 4.0), which permits any use, distribution and reproduction in any medium, provided the original author(s) and source are credited.

\section{References}

[1] Z. Nagy and D.E. Soper, A parton shower based on factorization of the quantum density matrix, JHEP 06 (2014) 097 [arXiv: 1401.6364] [INSPIRE].

[2] The code is available at Deductor: a parton shower event generator webpage, http://www.desy.de/ znagy/deductor and http://pages.uoregon.edu/soper/deductor/.

[3] Z. Nagy and D.E. Soper, Parton showers with quantum interference, JHEP 09 (2007) 114 [arXiv: 0706.0017] [INSPIRE].

[4] Z. Nagy and D.E. Soper, Parton showers with quantum interference: leading color, spin averaged, JHEP 03 (2008) 030 [arXiv:0801.1917] [INSPIRE].

[5] Z. Nagy and D.E. Soper, Parton showers with quantum interference: leading color, with spin, JHEP 07 (2008) 025 [arXiv:0805.0216] [INSPIRE].

[6] Z. Nagy and D.E. Soper, Parton shower evolution with subleading color, JHEP 06 (2012) 044 [arXiv: 1202.4496] [INSPIRE].

[7] Z. Nagy and D.E. Soper, Parton distribution functions in the context of parton showers, arXiv: 1401.6368 [INSPIRE].

[8] G. Marchesini et al., HERWIG: a Monte Carlo event generator for simulating hadron emission reactions with interfering gluons. Version 5.1 - April 1991, Comput. Phys. Commun. 67 (1992) 465 [INSPIRE].

[9] M. Bahr et al., HERWIG++ physics and manual, Eur. Phys. J. C 58 (2008) 639 [arXiv: 0803.0883] [INSPIRE].

[10] T. Sjöstrand, High-energy physics event generation with PYTHIA 5.7 and JETSET 7.4, Comput. Phys. Commun. 82 (1994) 74 [InSPIRE].

[11] T. Sjöstrand, S. Mrenna and P.Z. Skands, A brief introduction to PYTHIA 8.1, Comput. Phys. Commun. 178 (2008) 852 [arXiv:0710.3820] [INSPIRE].

[12] T. Gleisberg et al., Event generation with SHERPA 1.1, JHEP 02 (2009) 007 [arXiv: 0811.4622] [INSPIRE].

[13] S. Schumann and F. Krauss, A parton shower algorithm based on Catani-Seymour dipole factorisation, JHEP 03 (2008) 038 [arXiv:0709.1027] [INSPIRE]. 
[14] G. Gustafson, Dual description of a confined color field, Phys. Lett. B 175 (1986) 453 [INSPIRE].

[15] G. Gustafson and U. Pettersson, Dipole formulation of QCD cascades, Nucl. Phys. B 306 (1988) 746 [INSPIRE].

[16] B. Andersson, G. Gustafson and L. Lönnblad, Gluon splitting in the color dipole cascades, Nucl. Phys. B 339 (1990) 393 [inSPIRE].

[17] L. Lönnblad, ARIADNE version 4: a program for simulation of QCD cascades implementing the color dipole model, Comput. Phys. Commun. 71 (1992) 15 [INSPIRE].

[18] W.T. Giele, D.A. Kosower and P.Z. Skands, A simple shower and matching algorithm, Phys. Rev. D 78 (2008) 014026 [arXiv:0707.3652] [InSPIRE].

[19] G. Marchesini and B.R. Webber, Simulation of QCD jets including soft gluon interference, Nucl. Phys. B 238 (1984) 1 [inSPIRE].

[20] R.K. Ellis, G. Marchesini and B.R. Webber, Soft radiation in parton parton scattering, Nucl. Phys. B 286 (1987) 643 [Erratum ibid. B 294 (1987) 1180] [INSPIRE].

[21] S.D. Ellis and D.E. Soper, Successive combination jet algorithm for hadron collisions, Phys. Rev. D 48 (1993) 3160 [hep-ph/9305266] [InSPIRE].

[22] S. Catani, Y.L. Dokshitzer, M.H. Seymour and B.R. Webber, Longitudinally invariant $K_{t}$ clustering algorithms for hadron hadron collisions, Nucl. Phys. B 406 (1993) 187 [InSPIRE].

[23] Z. Nagy and D.E. Soper, Matching parton showers to NLO computations, JHEP 10 (2005) 024 [hep-ph/0503053] [INSPIRE].

[24] Z. Nagy and D.E. Soper, A new parton shower algorithm: shower evolution, matching at leading and next-to-leading order level, in Ringberg workshop on new trends in HERA physics 2005, G. Grindhammer et al. eds., World Scientific, Singapore (2006) [hep-ph/0601021] [INSPIRE].

[25] S. Catani and M.H. Seymour, A general algorithm for calculating jet cross-sections in NLO QCD, Nucl. Phys. B 485 (1997) 291 [Erratum ibid. B 510 (1998) 503] [hep-ph/9605323] [INSPIRE].

[26] S. Platzer and S. Gieseke, Coherent parton showers with local recoils, JHEP 01 (2011) 024 [arXiv:0909.5593] [INSPIRE].

[27] M. Dinsdale, M. Ternick and S. Weinzierl, Parton showers from the dipole formalism, Phys. Rev. D 76 (2007) 094003 [arXiv: 0709.1026] [INSPIRE].

[28] T. Sjöstrand and P.Z. Skands, Transverse-momentum-ordered showers and interleaved multiple interactions, Eur. Phys. J. C 39 (2005) 129 [hep-ph/0408302] [INSPIRE].

[29] N. Fischer, S. Gieseke, S. Plätzer and P. Skands, Revisiting radiation patterns in $e^{+} e^{-}$ collisions, Eur. Phys. J. C 74 (2014) 2831 [arXiv:1402.3186] [INSPIRE].

[30] V.N. Gribov and L.N. Lipatov, Deep inelastic ep scattering in perturbation theory, Sov. J. Nucl. Phys. 15 (1972) 438 [Yad. Fiz. 15 (1972) 781] [inSPIRE].

[31] G. Altarelli and G. Parisi, Asymptotic freedom in parton language, Nucl. Phys. B 126 (1977) 298 [INSPIRE].

[32] Y.L. Dokshitzer, Calculation of the structure functions for deep inelastic scattering and $e^{+} e^{-}$ annihilation by perturbation theory in quantum chromodynamics., Sov. Phys. JETP 46 (1977) 641 [Zh. Eksp. Teor. Fiz. 73 (1977) 1216] [INSPIRE]. 
[33] Y. Dokshitzer and G. Marchesini, Monte Carlo and large angle gluon radiation, JHEP 03 (2009) 117 [arXiv: 0809.1749] [INSPIRE].

[34] Z. Nagy and D.E. Soper, Final state dipole showers and the DGLAP equation, JHEP 05 (2009) 088 [arXiv:0901.3587] [INSPIRE].

[35] P.Z. Skands and S. Weinzierl, Some remarks on dipole showers and the DGLAP equation, Phys. Rev. D 79 (2009) 074021 [arXiv: 0903.2150] [inSPIRE].

[36] J.C. Collins, D.E. Soper and G.F. Sterman, Transverse momentum distribution in Drell-Yan pair and $W$ and $Z$ boson production, Nucl. Phys. B 250 (1985) 199 [INSPIRE].

[37] Z. Nagy and D.E. Soper, On the transverse momentum in Z-boson production in a virtuality ordered parton shower, JHEP 03 (2010) 097 [arXiv:0912.4534] [INSPIRE].

[38] S. Bethke, Z. Kunszt, D.E. Soper and W.J. Stirling, New jet cluster algorithms: next-to-leading order QCD and hadronization corrections, Nucl. Phys. B 370 (1992) 310 [Erratum ibid. B $\mathbf{5 2 3}$ (1998) 681] [INSPIRE].

[39] V.S. Fadin, E.A. Kuraev and L.N. Lipatov, On the Pomeranchuk singularity in asymptotically free theories, Phys. Lett. B 60 (1975) 50 [inSPIRE].

[40] E.A. Kuraev, L.N. Lipatov and V.S. Fadin, Multi-Reggeon processes in the Yang-Mills theory, Sov. Phys. JETP 44 (1976) 443 [Erratum ibid. 45 (1977) 199] [Zh. Eksp. Teor. Fiz. 71 (1976) 840] [INSPIRE].

[41] E.A. Kuraev, L.N. Lipatov and V.S. Fadin, The Pomeranchuk singularity in non-Abelian gauge theories, Sov. Phys. JETP 45 (1977) 199 [Zh. Eksp. Teor. Fiz. 72 (1977) 377] [INSPIRE].

[42] I.I. Balitsky and L.N. Lipatov, The Pomeranchuk singularity in quantum chromodynamics, Sov. J. Nucl. Phys. 28 (1978) 822 [Yad. Fiz. 28 (1978) 1597] [InSPIRE].

[43] C.R. Schmidt, A Monte Carlo solution to the BFKL equation, Phys. Rev. Lett. 78 (1997) 4531 [hep-ph/9612454] [INSPIRE].

[44] L.H. Orr and W.J. Stirling, Dijet production at hadron hadron colliders in the BFKL approach, Phys. Rev. D 56 (1997) 5875 [hep-ph/9706529] [INSPIRE].

[45] H. Jung and G.P. Salam, Hadronic final state predictions from CCFM: the hadron level Monte Carlo generator CASCADE, Eur. Phys. J. C 19 (2001) 351 [hep-ph/0012143] [INSPIRE].

[46] H. Jung et al., The CCFM Monte Carlo generator CASCADE version 2.2.03, Eur. Phys. J. C 70 (2010) 1237 [arXiv:1008.0152] [INSPIRE].

[47] J.R. Andersen and J.M. Smillie, Multiple jets at the LHC with high energy jets, JHEP 06 (2011) 010 [arXiv:1101.5394] [InSPIRE].

[48] M. Slawinska, S. Jadach and K. Kutak, A new Monte Carlo study of evolution equation with coherence, Phys. Lett. B 722 (2013) 151 [arXiv:1302.0293] [InSPIRE].

[49] M. Cacciari, G.P. Salam and G. Soyez, The anti- $k_{T}$ jet clustering algorithm, JHEP 04 (2008) 063 [arXiv:0802.1189] [INSPIRE].

[50] M. Cacciari, G.P. Salam and G. Soyez, FastJet user manual, Eur. Phys. J. C 72 (2012) 1896 [arXiv:1111.6097] [INSPIRE]. 